\title{
Attività sismica in Italia nel 1969
}

(Seismic activity in Italy in 1969)

\section{Gasparini (*)}

Ricevuto il 19 Marzo 1974

\begin{abstract}
Riassunto. - Sono stati raccolti ed elaborati tutti i dati riguardanti gli eventi sismici che hanno interessato in qualche molo l'Italia nel 1969. I terremoti sono stati riportati in un clenco e per quelli di maggior interesse, è stata fatta una descrizione particolareggiata, con alcuni accenni alla tettonica della zona epicentrale. Quando è stato possibile sono state tracciate le isosiste e si d̀ indagato sulla natura del movimento all'ipocentro. In ultimo sono stati riportati alcuni eventi sismici con epicentro lontano che per la loro particolare intensità hanno interessato anche l'Italia.
\end{abstract}

SUMMARY. - All data coneerning the seismic events which in some way interested Italy in 1969 have been gathered and carefully drawn up. The earthquakes have been listed, with a detailed deseription provided for those of major interest indicating the tectonics of the epicenter zone. When it was possible, the isoseismals were traced and research was made on the earthquake mechanism. Listed at the end are the seismic events with far away epicenters which, for their particular intensity, nevertheless concern Italy.

PREMTSSA

Questo lavoro rispecchia in linea generale quello già pubblicato per gli eventi del $1970\left({ }^{14}\right)$. Si è ritenuto adottare la stessa simbologia onde conservare un'omogeneità agli elenchi degli eventi sismici ed

(*) Osservatorio Sismico Centrale M. Porzio Catone - Istituto Nazionale di Geofisica, Roma. 
anche per non creare inutili confusioni. Per una maggiore rapiclità di consultazione si riporta appresso un elenco della simbologia usata ed i) suo significato pratico. Per eventuali maggiori chiarimenti si rimanda al precedente articolo ed alla Monografia sui fenomeni dell'Anconitano ${ }^{(9)}$, oltreché alla bibliografia.

\section{Simbologia adottata.}

Att. $\quad-$ Attendibilità dei dati riportati nell'elenco, il primo numero si riferisce alle coordinate epicentrali, il secondo alla profondità, il terzo alla magnitudo.

Dis. - Numero relativo al distretto sismotettonico in cui cade l'epicentro.

I) - Distanza ipocentrale in $\mathrm{km}$.

$I_{0} \quad$ - Intensità macrosismica all'epicentro in Mercalli-Sieberg.

I - Intensità macrosismica in Mercalli-Sieberg.

Località - Centro abitato maggiormente colpito e più vicino all'epicentro.

$M \quad$ - Magnitudo strumentale ricavata dalle semi ampiezze della fase massima sulle due componenti orizzontali a breve periodo.

$M^{*} \quad$ - Magnitudo macrosismica.

h - Profonditì ipocentrale in $\mathrm{km}$.

\%ona - Regione geografica in cui è compresa l'area megasismica.

$a$ - Coefficiente di attenuazione dell'energia macrosismica in superficie (per il percorso considerato).

$a$ - Coefficiente medio di attenuazione dell'energia macrosismica in superficie (per il percorso considerato).

$\Delta \quad$ - Distanza epicentrale in $\mathrm{km}$.

$\varphi \quad-$ Latitudine nord.

$\lambda$ - Longitudine est.

$H_{\mathrm{o}} \quad$ - Tempo origine riferito all'ora di Greenwich.

\section{DATI STATISTICI}

Nel 1969 dalla rete sismica dell'Istituto Nazionale di Geofisica, sono stati registrati 534 eventi avvenuti in Italia. Di questi: 36 sono stati provocati quasi certamente da esplosioni, 271 hanno dato luogo ad una chiara registrazione (ma solo per 79 di essi è stato possibile individuare l'epicentro), per gli altri 227 non è stata effettuata l'in- 
terpretazione completa del sismogramma data la debole intensitì e chiarezza della registrazione (tracce).

\section{1 - NUOVE RICERCHE}

1.1 - Magnitudo - Nel 1969 presso l'Osservatorio di Roma Monte Porzio non erano in funzione i sismometri Wood-Anderson per la determinazione della magnitudo, come dall'originaria idea di Richter ( $\left.{ }^{17}\right)$. Per colmare tale vuoto è stata effettuata una ricerca supplementare già pubblicata $\left({ }^{2}\right)$.

La relazione che ha permesso di ricavare la magnitudo è stata ottenuta tramite un confronto sperimentale tra le registrazioni sismiche effettuate dai Lehener-Griffith e dai Wood-Anderson contemporaneamente. Si è ricavata così una curva di correlazione tra i logaritmi delle semi ampiezze delle fasi massime in funzione della distanza epicentrale.

La relazione ottenuta è:

$$
c=-2,2 \tau \cdot 10^{-6} \Delta^{2}+9,05 \cdot 10^{-3} \Delta+0,184
$$

in cui $c$ è il valore di correzione da sommare o sottrarre al " $\log b$ " calcolato da Richter per il Wood-Anderson, $\Delta$ è la distanza epicentrale.

1.2 - Natura della scossa all'ipocentro - Per tre eventi sismici avvenuti nel 1969 è stato possibile indagare sulla natura della scossa all'ipocentro. Per questa ricerca è stato seguito il metodo di Byerly $\left({ }^{5,6,7}\right)$ esteso da Di Filippo (11,12), e con l'ausilio del calcolatore elettronico, in base ad un programma elaborato dal Dott. R. Console.

Il metodo seguito prevede che la frattura degli strati sia causata da una coppia di forze (principale) che dà luogo ad una distribuzione a quadranti dei versi delle onde $P$. La posizione dei piani nodali è stata determinata dall'elaboratore per tentativi, minimizzando un'opportuna funzione che tiene conto dell'accordo tra i versi registrati dalle stazioni e quelli previsti dalla teoria. Le posizioni teoriche dei piani nodali sia per le onde $P$ che per le $S$ per la coppia semplice, ed anche per altri tipi di forze non prese in considerazione in questo lavoro, sono ampiamente trattate da Keylis Borok ${ }^{(15)}$.

La rappresentazione in superficie del fenomeno è stata effettuata mediante la proiezione stereografica polare, sul cui piano equatoriale 
sono state proiettate le stazioni e l'intersezione tra i piani noclali e la superficie terrestre. Il centro della figura corrisponde all'ipocentro. Ia posizione delle stazioni, supposta la terra omogenea, corrisponde con il punto d'intersezione tra il raggio sismico rettificato e la sfera ipocentrale.

2 - Principali terremoti avvenuti veli'AnNo

\section{1 - 6 Gennaio Appennino toscano}

Dati epicentrali

$H_{\mathrm{o}}=22^{\mathrm{h}} 03^{\mathrm{m} 28^{\mathrm{s}}} ; \varphi=44^{\circ} 04^{\prime} \mathrm{N} \lambda=10^{\circ} 44^{\prime} \mathrm{E} ; h=20 \mathrm{~km} ; M=4,0$ (RMP)

$I_{0}=\mathrm{VI}$

Altre determinazioni epicentrali

USCGS $-H_{0}=22^{\mathrm{n}} 03^{\mathrm{m} 24,7 \mathrm{~s}} ; \varphi=44,1^{\circ} \mathrm{N} \quad \lambda=10,7^{\circ} \mathrm{E} ; h=7 \mathrm{~km} ;$ $M=4,5$ (CGS)

BCIS $-H_{0}=22^{\mathrm{n}} 03^{\mathrm{m} 25^{\mathrm{s}}} ; \varphi=44,0^{\circ} \mathrm{N} \quad \lambda=10,8^{\circ} \mathrm{E}$

MOS $-H_{0}=22^{\mathrm{h}} 03^{\mathrm{m} 29^{\mathrm{s}}} ; \quad \varphi=44,3^{\circ} \mathrm{N} \quad \lambda=10,7^{\circ} \mathrm{E}$

ISC $-H_{\mathrm{o}}=22^{\mathrm{n}} 03^{\mathrm{m}} 28,9^{\mathrm{s}} ; \varphi=44,14^{\circ} \mathrm{N} \quad \lambda=10,80^{\circ} \mathrm{E} ; h=33 \mathrm{~km} ;$ $M=4,1$

Notizie macrosismiche

I'area megasismica ha interessato l'Alta Val di Lima. Sono state raccolte le seguenti notizie macrosismiche:

VI a San Marcello Pistoiese, Popiglio, Cutigliano, San Cassiano.

V.VI a Lizzano, Piteglio, Bagni di Lucca, Abetone, Pian di Ortani.

V a Gavinana, Pontepetri, Piastre, Prunetta, Pistoia, Serra Pistoiese, Marliana, Serravalle Pistoiese, Monsummano Terme, Montecatini, Buggiano, Borgo a Mazzano, Gallicano, Molazzana.

IV a Tresana, Podenzana, Massa Carrara, Serravezza, Casoli, Massarosa, Ia Spezia, Viareggio, Pisa, Lucca, Capannori, Poggio a Caiano, Prato, Vaiano, Vernio, Frassinoro, Fivizzano. 


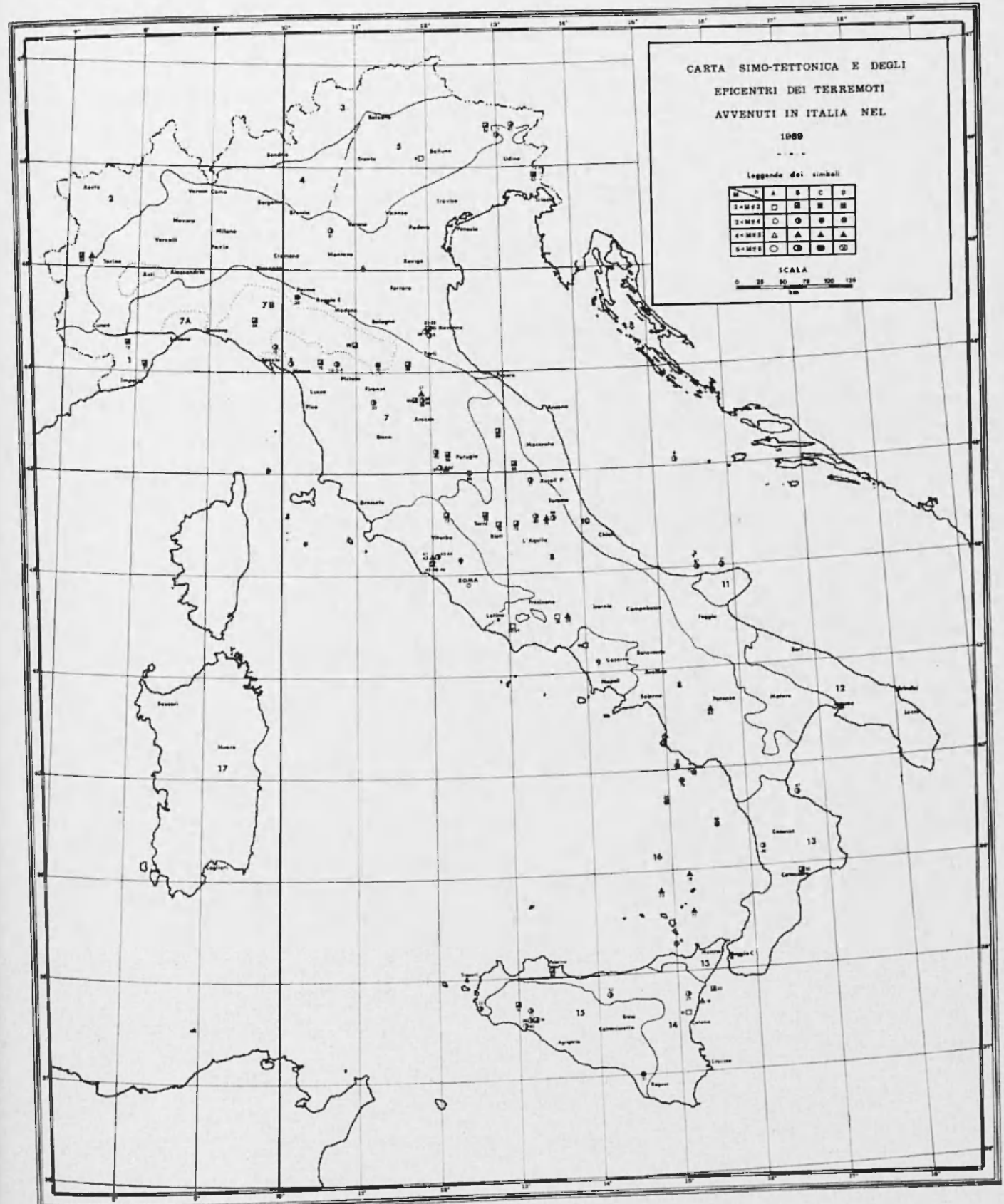

Fig. I 
III a Firenze, Empoli, Orentano, Altopascio, Crespina, Bagnone, Mulazzo, Sesta Godena, Chiavari, Riomaggiore.

Alla scossa principale sono seguite tre repliche:

$\begin{array}{llll}6 \text { Gennaio ore } 2219 & \dot{I}_{0}=\text { III } & M=3,2 & h=20 \\ 7 \text { Gennaio ore } 0155 & I_{0}=\text { IV } & M=3,6 & h=21 \\ 7 \text { Gennaio ore } 0228 & I_{0}=\text { IV } & M=3,6 & h=17\end{array}$

Talle isosiste, fig. 2, si è riscontrata una maggiore propagazione dell'energia macrosismica in Garfagnana e lungo la dorsale delle Apuane, fino ar interessare la parte occirlentale dell'Appennino Ligure costituito dal flysh cretacico. Fenomeni di attenuazione si hanno nel Frignano e lungo il versante Padano dell'Appennino Tosco-Emiliano, a sud uguale fenomeno si ha in corrispondenza delle formazioni mioplioceniche. L'Isosista di IV presenta un lobo di attenuazione in corrispondenza delle Ofioliti di Montemurlo.

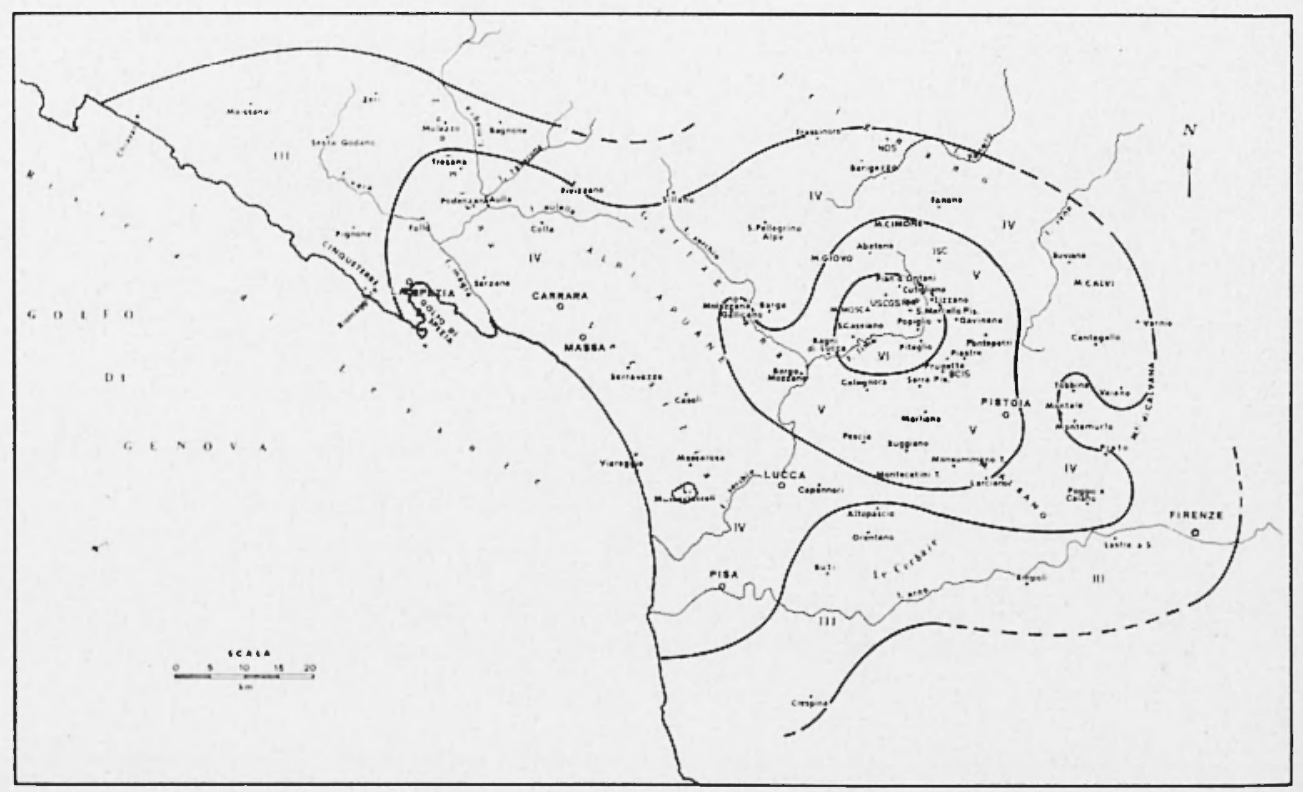

Fig. 2 - Isosiste del terremoto dell'Appennino toseano, 6 Gennaio 1969. 
Tabella 1 - Valori Del coefficiexte di attexuazione deli.'exergia MACROSISMICA IN SUPERFICIE.

\begin{tabular}{|c|c|c|}
\hline $\begin{array}{l}\text { Direzione dell'apicentro } \\
\text { percor'so nelle formazioni }\end{array}$ & $A$ & $\alpha \circ \bar{u}$ \\
\hline $\begin{array}{l}\text { Sud } \\
\text { - macigno, Verrucano, basamento } \\
\text { autoctono etrusco-ligure }\end{array}$ & 37 & $0,0250 \pm 0,0066$ \\
\hline $\begin{array}{l}\text { Nord } \\
\text { alloctone, complesso delle argille } \\
\text { scagliose, pliocene del margine } \\
\text { padano }\end{array}$ & 27 & $0,0355 \pm 0,0057$ \\
\hline Ovest-Nord Ovest & 15 & 0,0363 \\
\hline $\begin{array}{l}\text { - macimo, complesso delle argille } \\
\text { scagliose, facies infracretacea }\end{array}$ & 120 & $0,00685 \pm 0,00004$ \\
\hline Sud-Est & 36 & $0,0215 \pm 0,0024$ \\
\hline $\begin{array}{l}\text { - parallelamente al limite padano } \\
\text { della facies etrusco-ligure, com- } \\
\text { plesso delle argille scagliose }\end{array}$ & & \\
\hline
\end{tabular}

\section{Lineamenti tettonici}

Il terremoto ha interessato l'esteso rovesciamento della serie toscana che va da M. Orsaro alla Tal di Lima, cioè quella zona soggiacente alla tettonica trasversale dell'Appennino Settentrionale. Le direttrici trasversali si sarebbero formate in seguito a movimenti verticali e differenziali, durante e dopo la fase tettogenetica con sovrascorrimenti separati da zone avanscorse.

Parlando delle cause orogeniche che hanno dato forma alla linea Livorno-Sillaro, Bortolotti ( $\left.{ }^{3}\right)$ ipotizza che il basamento della serie toscana tende ad abbassarsi nella zona a nord di detta linea e che questa sia situata in una zona di flessura o in corrispondenza di un sistema di faglie normali immergenti a Nord.

L'analisi dell'evoluzione di tale linea nelle varie ere geologiche porterebbe a ritenere che essa è ancora attiva, ed è causa di molti eventi sismici.

Nello studio del terremoto della Garfagnana 1939, Rosini (18) ricavò come natura della scossa un sollevamento degli strati. 
L'ipocentro dell'attuale sisma situato a $20 \mathrm{~km}$, verrebbe ad interessare lo strato del granito. Il meccanismo all'ipocentro che ha provocato l'attuale scossa è risultato di frattura.

\section{Natura della scossa all'ipocentro}

Ia polarità degli arrivi delle onde $P$ nei vari osservatori ha permesso di calcolare la natura della scossa all'ipocentro. Sono state usate per tale ricerca 11 stazioni le cui polarità sono risultate tutte compatibili con il morlello teorico. L'esigno numero di stazioni che sono risultate utilizzabili, rende meno attendibili i risultati ottenuti, purtuttavia essi possono essere considerati accettabili per un'indicazione generale del fenomeno tellurico.

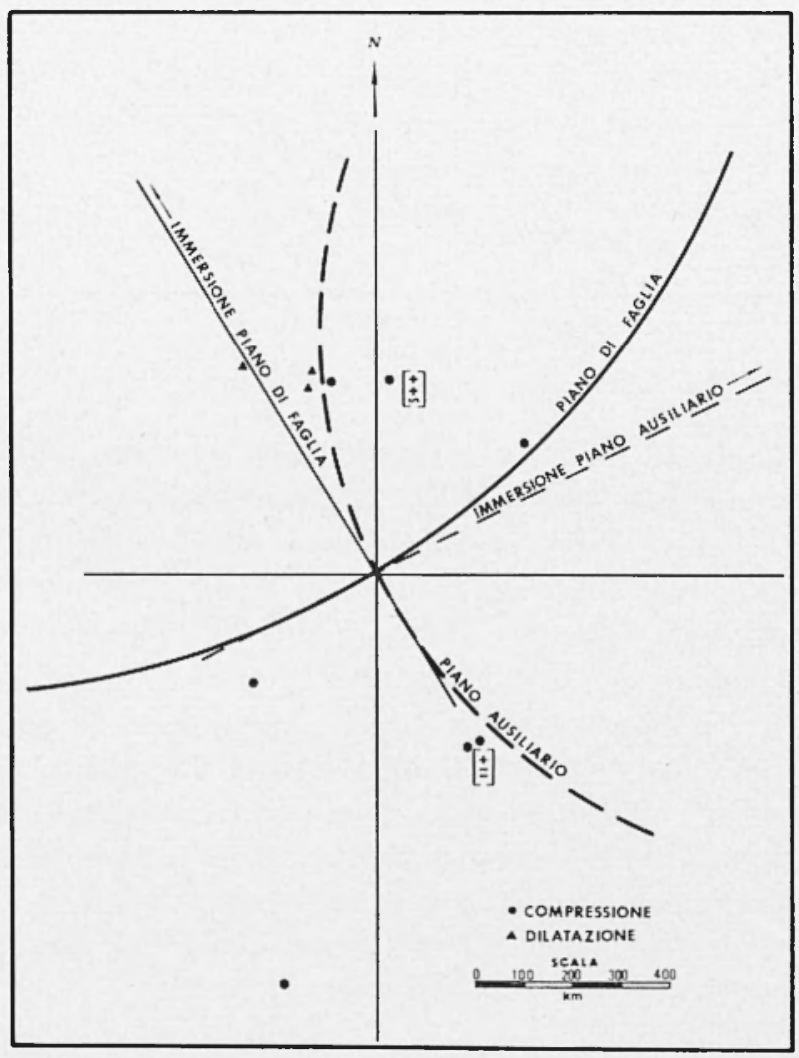

Fig. 3 - Proiezione stereografica polare del meccanismo allipocentro, terremoto del 6 Gennaio 1969, Appennino toseano. 
La distribuzione dei primi impulsi delle $P$ (fig. 3) assegnano al primo piano nodale direzione NO-SE con un'inclinazione di $74^{\circ}$ eirca verso NE. Il secondo piano nodale ha direzione circa NE-SO, inclinato di $80^{\circ}$ circa verso NO. L'analisi dei primi impulsi delle $S$ indicano come piano di faglia il secondo piano nodale delle $P$.

Tali risultati si accortano con quelli geologici.

Dati epicentrali

$H_{0}=16^{\mathrm{h}} 17^{\mathrm{m}} 32^{\mathrm{s}} ; p=44^{\circ} 23^{\prime} \mathrm{N} \lambda=12^{\circ} 00^{\prime} \mathrm{E} ; h=15 \mathrm{~km} ; M=3,9$ (RMP) $I_{0}=\mathrm{V}$

Altre determinazioni epicentrali

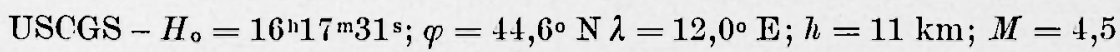
(CGS)

BCIS $-H_{\mathrm{o}}=16^{\mathrm{h}} 17^{\mathrm{m}} 33^{\mathrm{s}} ; \varphi=44,5^{\circ} \mathrm{N} \lambda=12,0^{\circ} \mathrm{E}$;

ISC $-H_{\mathrm{o}}=16^{11} 17^{\mathrm{m}} 32 \mathrm{~s} ; \varphi=44,5^{\circ} \mathrm{N} \lambda=12,1^{\circ} \mathrm{E} ; \pi=22 \pm 14 \mathrm{~km}$; $M=4,4$

Notizie macrosismiche

Il terremoto è stato sentito di:

V a Bagnacavallo, Russi, Cotignola.

IV a Forli, Faenza, Solarolo, Lugo di Romagna, Ravenna.

III a Brisighella, Modligliana, Dovadola, Alfonsine, S. Agata sul Santerno.

II a Cesena, Poggio Berni, Gatteo, Sarsina, Casal Valsenio.

L'andamento circolare delle isosiste, fig. 4, dimostrano una pressoché uniforme propagazione dell'energia sismica nelle varie direzioni. Il ravvicinamento maggiore che esse presentano verso la Valle Padana è dovuto all'attenuazione causata dalla coltre sedimentaria. I valori del coefficiente di attenuazione in superficie sono riportati in tabella 2. 
'TABELLA 2

\begin{tabular}{|c|c|c|}
\hline $\begin{array}{l}\text { Direzione dallepicentro } \\
\text { percorso nelle formazioni }\end{array}$ & .1 & $\alpha \circ \bar{a}$ \\
\hline $\begin{array}{l}\text { Nord } \\
\text { alluvionali con potenza media di } \\
4000 \mathrm{~m} \text { fino alla base del pliocene }\end{array}$ & 16 & $0,0733 \pm 0,0003$ \\
\hline $\begin{array}{l}\text { sud } \\
\text { _- della fascia bordiera padana, per- } \\
\text { pendicolarmente alla medesima }\end{array}$ & 32 & $0,0245 \pm 0,0003$ \\
\hline $\begin{array}{l}\text { Nord-Est } \\
\text { plio-quaternarie con potenza me- } \\
\text { dia di } 2000 \mathrm{~m} \text {, parallelamente alla } \\
\text { fascia bordiera padinal }\end{array}$ & 21 & $0,0414 \pm 0,0054$ \\
\hline $\begin{array}{l}\text { Sud-ovest } \\
\text { - plio-quaternarie con potenza me- } \\
\text { dia di jooo m. Tungo l'asse della } \\
\text { sinclinale romagnola }\end{array}$ & 27 & $0,0291 \pm 0,0027$ \\
\hline
\end{tabular}

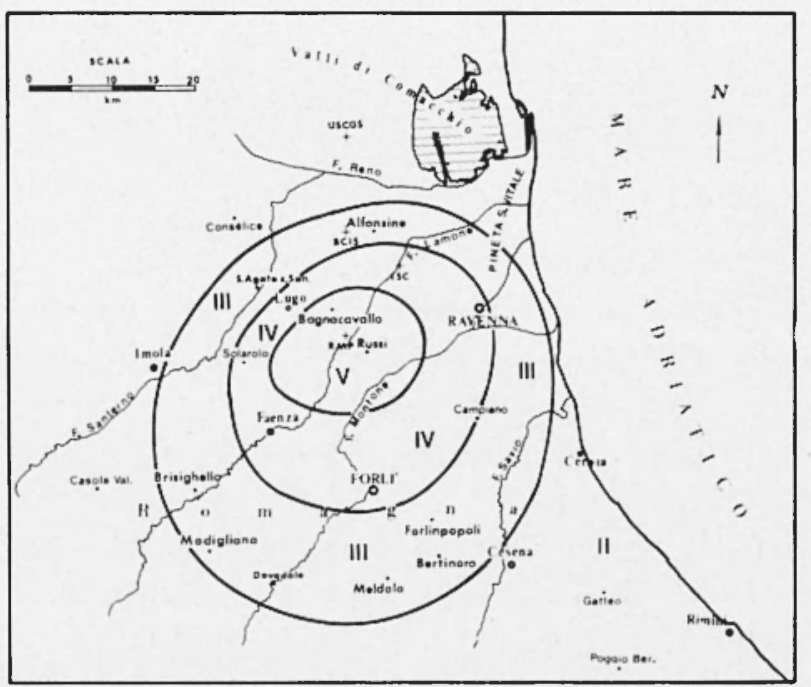

Fig. 4 - Isosiste del terremoto di Romagna, 10 Gennaio 1969. 
La rlebole potenza rlello strato del granito e relle formazioni geologiche antiche fanno assumere alle isoanomale di Bonguer un minimo negativo lungo tutta la fascia bordiera parlana, versante $\Lambda$ ppenninico. L'ipocentro posto a $15 \mathrm{~km}$, verrebbe arl interessare cosi la prima discontinuità della crosta che in questa zona dovrebbe essere intorno ai $15-20 \mathrm{~km}$ di profondità.

$2.3-29$ MLARzo

Costa del Cilento

Dati epicentrali

$H_{\mathrm{o}}=01^{\mathrm{h}} 43^{\mathrm{m}} 38^{\mathrm{s}} ; \varphi=40^{\circ} 02^{\prime} \mathrm{N} \lambda=15^{\circ} 06^{\prime} \mathrm{E} ; h=310 \mathrm{~km} ; M=4,0(\mathrm{RMIP})$

Altre determinazioni epicentrali

USCGS $-H_{0}=01^{\mathrm{h}} 43^{\mathrm{m}} 39^{\mathrm{s}} ; \varphi=40,0^{\circ} \mathrm{N} \lambda=15,2^{\circ} \mathrm{E} ; \not=310 ; M=4,6$ (CGS)

BCIS $-H_{\mathrm{o}}=01^{\mathrm{n}}+3^{\mathrm{m}} 38^{\mathrm{s}} ; \varphi=39,9^{\circ} \mathrm{N} \lambda=15,1^{\circ} \mathrm{E} ; h=320$

$\operatorname{MOS}-H_{\mathrm{o}}=01^{\mathrm{h}} 43^{\mathrm{m}} 44^{\mathrm{s}} ; \phi=40,4^{\circ} \mathrm{N} \lambda=15,3^{\circ} \mathrm{E} ; h=350$

ISC $\quad-H_{\mathrm{o}}=01^{\mathrm{h}} 43^{\mathrm{m}} 38^{\mathrm{s}} ; \varphi=40,0^{\circ} \mathrm{N} \lambda=15,1^{\circ} \mathrm{E} ; h=310 ; M=4,6$

Natura della scossa all'ipocentro

E stato possibile individuare le cause che hanno determinato questo terremoto tramite l'analisi delle compressioni e dilatazioni che l'onda sismica ha trasmesso al suolo nei vari osservatori.

Sono state raccolte le polarità dei primi impulsi in 34 stazioni, delle quali però 7 hanno dato un verso errato rispetto alla soluzione più probabile. L'errore risultante del $21 \%$ ì sufficientemente accettabile. Delle rimanenti 27 stazioni, 11 hanno dato polariti positiva le altre 16 polarità negativa. Nella fig. 5 sono riportate in proiezione stereografica polare le posizioni dei vari osservatori con la rispettiva polarità e la posizione dei piani norlali delle $P$, uno dei quali corrisponde al piano di faglia.

Ia soluzione più probabile per i due piani norlali laa $\mathrm{i}$ seguenti valori:

- per il piano $\Lambda$, immersione $\mathrm{E} 80,8^{\circ} \mathrm{S}$, inclinazione $27,8^{\circ}$

- per il piano $\mathrm{B}$, immersione $\mathrm{N} 73,6^{\circ} \mathrm{E}$, inclinazione $86,0^{\circ}$. 


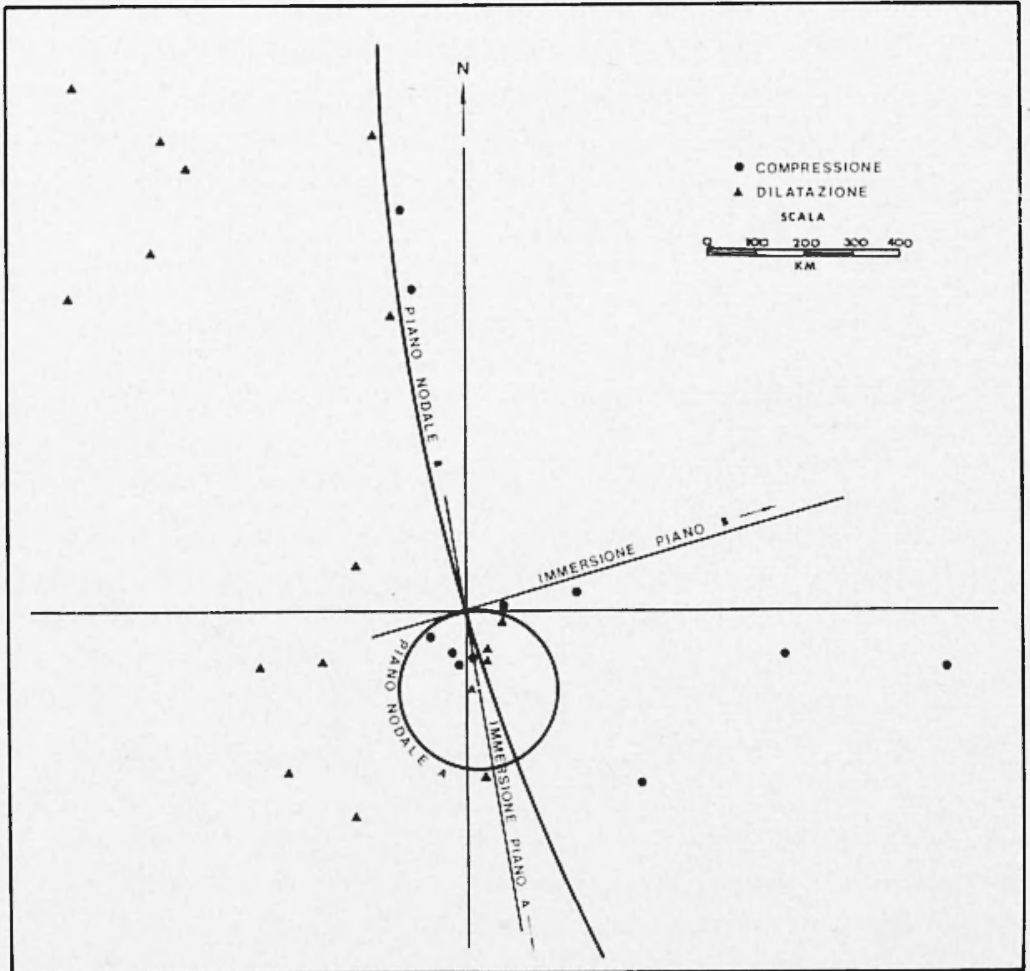

Fig. 5 - Proiezione stereografica polare del meccanismo all'ipocentro, terremoto del 29 Mar\%o 1969, epicentro nei pressi della costa cilentina.

$2.1-2$ APRILE Bisso TIRRENo

\section{Dati epicentrali}

$H_{\mathrm{o}}=01^{1{ }^{1}} 38^{\mathrm{m} 01 \mathrm{~s}} ; \varphi=38^{\circ} 58^{\prime} \mathrm{N} \lambda=15^{\circ} 14^{\prime} \mathrm{E} ; h=263 \mathrm{~km} ; M=4,8(\mathrm{RMP})$

Altre determinazioni epicentrali

$$
\begin{aligned}
& \text { USCGS }-H_{\mathrm{o}}=01^{\mathrm{n}} 38^{\mathrm{m}} 02^{\mathrm{s}} ; \varphi=39,0^{\circ} \mathrm{N} \lambda=15,3^{\circ} \mathrm{E} ; h=258 ; M=4,8 \\
& \quad(\mathrm{CGS}) \\
& \text { BCIS }-H_{0}=01^{\mathrm{h}} 38^{\mathrm{m}} 01^{\mathrm{s}} ; \varphi=38,9^{\circ} \mathrm{N} \lambda=15,3^{\circ} \mathrm{E} ; h=275 \\
& \text { MOS }-H_{0}=01^{1} 38^{\mathrm{m}} 01^{\mathrm{s}} ; \varphi=39,5^{\mathrm{o}} \mathrm{N} \lambda=15,4^{\circ} \mathrm{E} ; h=320 \\
& \text { ISC }-H_{\mathrm{o}}=01^{{ }^{1} 38^{\mathrm{m}} 02^{\mathrm{s}}} ; \varphi=39,0^{\circ} \mathrm{N} \lambda=15,2 \circ \mathrm{E} ; h=263 ; M=4,7
\end{aligned}
$$




\section{NTatura della scossa all'ipocentro}

In 64 stazioni è stato possibile individuare le polarità dei primi impulsi, delle quali sono risultate 25 compressioni e 39 dilatazioni.

Dalla ricerca analitica della soluzione più probabile è risultato che 10 stazioni hanno dato una polarità errata, cioè non compatibile con la migliore soluzione teorica. L'errore del $15 \%$ rientra ampiamente nei limiti di accettabilità. Nella fig. 6 sono riportati in proiezione ste-

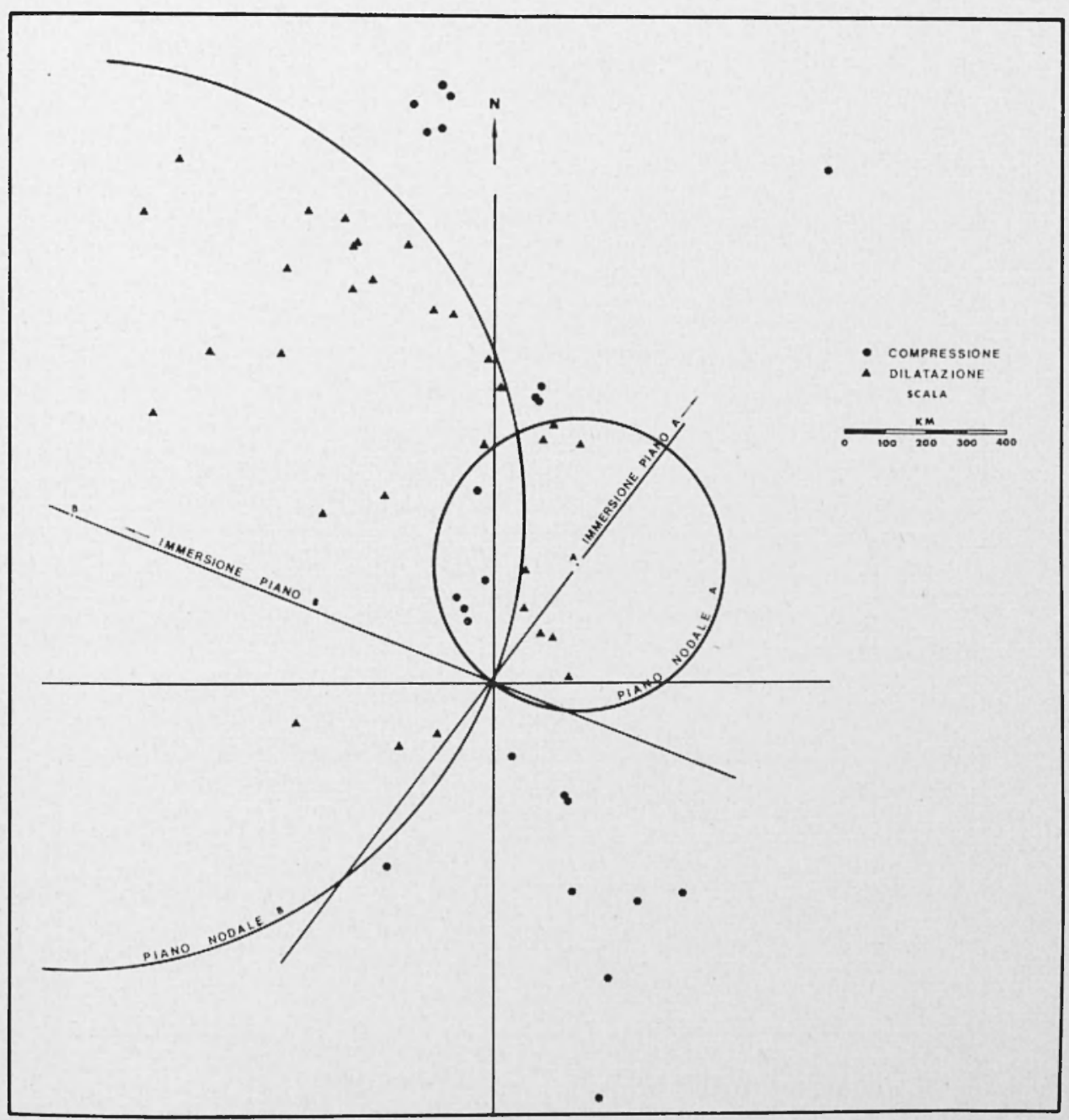

Fig. 6 - Proiezione stereografica polare del meccanismo all'ipocentro, sisma del basso Tirreno, 2 Aprile 1969. 
reografica polare le posizioni delle stazioni, i piani nodali e la loro immersione. Nel calcolo per la ricerca della soluzione probabile sono state poi escluse le stazioni che hanno dato polarità errata.

I valori ottenuti per l'individuazione del piano di faglia sono:

- piano nodale $A$, immersione $\mathrm{N} 36,1^{\circ} \mathrm{E}$, inclinazione $48,8^{\circ}$

- piano nodale $\mathrm{B}$, immersione $\mathrm{N} 68,3^{\circ} \mathrm{O}$, inclinazione $74,2^{\circ}$.

$2.5-16$ APRILE

ROMLANA

Jati epicentrali

$H_{o}=21^{\mathrm{n} 2} 23^{\mathrm{m}} 30^{\mathrm{s}} ; \varphi=44^{\circ} 25^{\prime} \mathrm{N} \lambda=11^{\circ} 58^{\prime} \mathrm{E} ; h=6 \mathrm{~km} ; M^{*}=3,5 ; I_{\mathrm{o}}=\mathrm{V}$

Notizie macrosismiche

Il terremoto è stato sentito di:

$\mathrm{V}$ a Bagnacavallo

IV a Lugo di Romagna, Fusignano

III a S. Agata sul Santerno, Massa Lombarda, Cotignola, Alfonsine II a Solaro, Faenza, Ravenna, Forli, Conselice.

La scossa principale è stata seguita da tre repliche, tab. 3 , i cui epicentri si possono considerare coincidenti con quello della scossa principale.

Tabella 3

\begin{tabular}{|c|c|c|c|c|}
\hline Data & Ore & $I_{0}$ & $1 I^{*}$ & $\hbar$ \\
\hline $17-\mathrm{IV}$ & 0816 & III-IV & 2,8 & 6 \\
$17-\mathrm{IV}$ & 0837 & III & 2,6 & 6 \\
$17-\mathrm{IV}$ & 1424 & III & 2,6 & 6 \\
\hline
\end{tabular}

L'andamento delle isosiste è quello consueto per i terremoti del Ravennate. La piccola profondità ipocentrale e la debole intensità sismica caratterizzano questo terremoto come causato da movimenti 


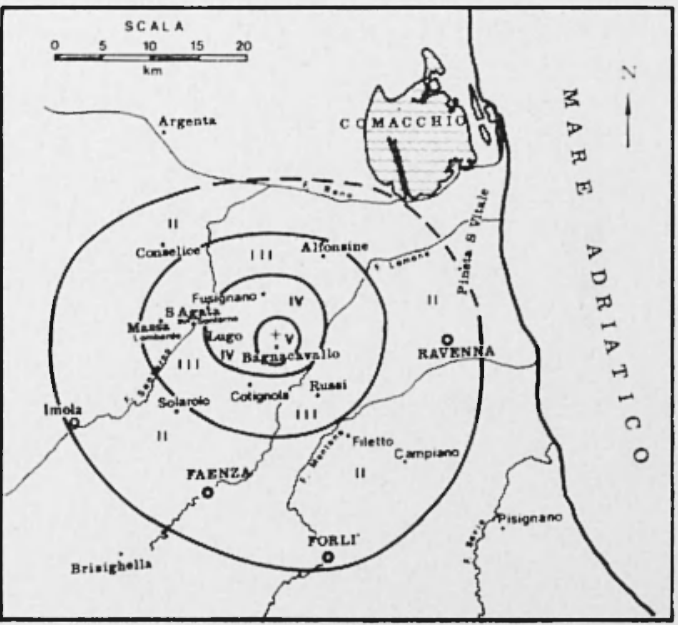

I'ig. 7 - Isosiste del terremoto del ravennate, 16 Aprile 1969.

negli strati superficiali della crosta. I coefficienti d'attenuazione dell'energia macrosismica in superficie sono riportati in tab. 4. In fig. 7 sono tracciate le isosiste di questo terremoto.

Tabelda 4

\begin{tabular}{|c|c|c|}
\hline $\begin{array}{l}\text { Direzione dall'epicentro } \\
\text { percorso nelle formazioni }\end{array}$ & $d$ & $a \circ \bar{a}$ \\
\hline $\begin{array}{l}\text { Nord-Est } \\
\text { - perpendicolarmente alle strutture } \\
\text { tettoniche sepolte. Potenza me- } \\
\text { dia dei sedimenti alla base del } \\
\text { Pliocene } 3000 \mathrm{~m} \text {. }\end{array}$ & 20 & $0,0762 \pm 0,0090$ \\
\hline Sud-Ovest & 5 & 0,1232 \\
\hline $\begin{array}{l}\text { - perpendicolarmente alla fascia } \\
\text { bordiera. Potenza media dei se- } \\
\text { dimenti } 2000 \mathrm{~m} \text { (alla base del } \\
\text { Pliocene) }\end{array}$ & 23 & $0,0598 \pm 0,0083$ \\
\hline Sud-Est & 5 & 0,14873 \\
\hline $\begin{array}{l}\text { - parallelamente alle strutture tet- } \\
\text { toniche sepolte. Potenza media } \\
\text { dei sedimenti } 1500 \mathrm{~m} \text { (alla base } \\
\text { del Pliocene) }\end{array}$ & 11 & $\begin{array}{l}0,0775 \\
0,0488\end{array}$ \\
\hline
\end{tabular}


Considerazioni tettoniche

L'epicentro localizzato a $1,5 \mathrm{~km}$ a Nord di Bagnacavallo, interessa i terreni argillosi-alluvionali del Ravennate. In tale zona la base del pliocene raggiunge i $3000 \mathrm{~m}$ e l'epicentro carlrebbe sul versante meridionale della sinclinale di Ravenna. Si puó presumere che il sisma sia stato causato da movimenti di faglie interessanti le formazioni mioceniche.

\section{Dati epicentrali}

$H_{0}=09^{\mathrm{h} 12^{\mathrm{m}} 29^{\mathrm{s}}} ; \varphi=41^{\circ} 35^{\prime} \times \lambda=13^{\circ} 47^{\prime} \mathrm{E} ; h=6 \mathrm{~km} ; M=4,6(\mathrm{RCU}) ;$ $I_{0}=$ VI-VII

Altri dati epicentrali

$$
\begin{aligned}
\text { USCGS }-H_{0} & =09^{\mathrm{h}} 12^{\mathrm{m} 29 \mathrm{~s}} ; \varphi=41,6^{\circ} \mathrm{N} \lambda=13,8^{\circ} \mathrm{E} ; h=6 \mathrm{~km} ; \\
H & =4,6 \\
\text { BCIS }-H_{\mathrm{o}} & =09^{\mathrm{n}} 12^{\mathrm{m}} 33^{\mathrm{s}} ; \varphi=41,5^{\circ} \mathrm{N} \lambda=13,7^{\circ} \mathrm{E} \\
\text { ISC }-H_{\mathrm{o}} & =09^{\mathrm{h}} 12^{\mathrm{m}} 34^{\mathrm{s}} ; \varphi=41,4^{\circ} \mathrm{N} \lambda=13,6^{\circ} \mathrm{E} ; h=40 \pm 24 \mathrm{~km} ; \\
H & =4,6
\end{aligned}
$$

\section{Notizie macrosismiche}

Il terremoto è stato sentito di:

VI-VII a Terelle

VI a S. Elia Fiumerapido, Belmonte Castello

V a Colle S. Miagno, Castrocielo, Cassino, Piedimonte

IV a Arpino, Casalettico, Atina, Roccasecca, Portella

III a Arce, Fontana Liri, Vallerotonda, Aquino

II a Frosinone, Pontecorvo, S. Giorgio al Liri, Sora, Picinisco, Cervaro.

L'attuale sisma presenta irlentiche caratteristiche agli innumerevoli eventi che già in passato colpirono la Ciociaria ed il Matese. Gli eventi generalmente superficiali si manifestano nei casi ri rilievo, in maniera distruttiva all'epicentro e si attenuano totalmente in un breve raggio di $15 \mathrm{~km}$ circa. 
Dalle isosiste, fig. 8, si nota che la direzione di massima propagazione è quella appenninica. Il sisma non si è sentito oltre la Valle del Sacco-Liri a SO, ad Est l'evento si è esaurito in corrispondenza clella direttrice meridiana delle Mainarde. L'isosista di II grado, a Nord, termina in corrispondenza delle propagini meridionali degli Ernici e della Marsica. In breve il sisma ha interessato esclusivamente la massiccia struttura calcarea di M. Cairo.

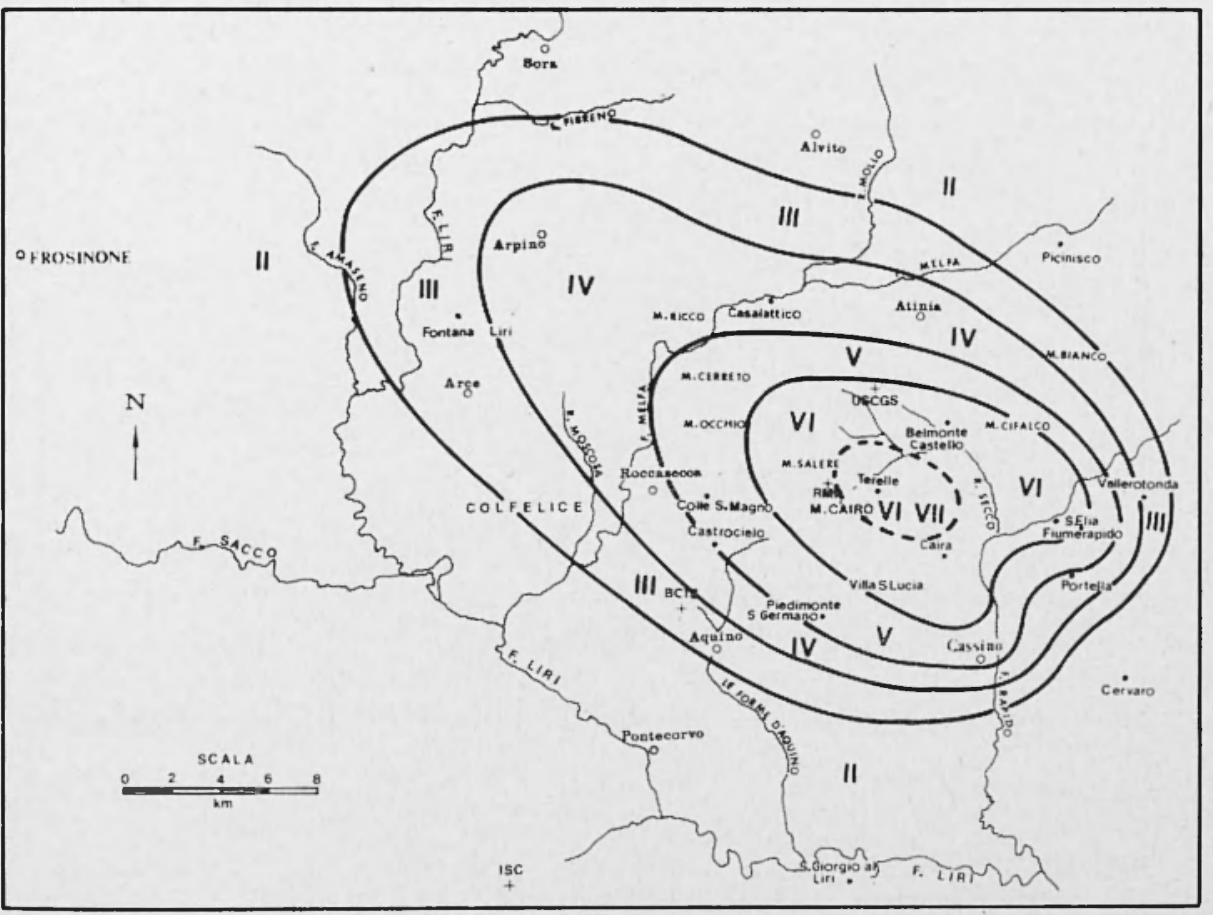

Fig. 8 - Isosiste del terremoto della bassa Ciociaria, 17 Aprile 1969.

\section{Lineamenti tettonici}

L'area colpita dal sisma è interamente compresa nella zona in "facies di piattaforma " ${ }^{(8)}$ costituita per lo più da fanghi calcarei. La potenza dei sedimenti dal calcare liassico alle arenarie tortoniane è di $3500 \mathrm{~m}$.

Il massiccio gruppo di M. Cairo è separato nella parte meridionale, verso la Valle Latina, da una faglia rettilinea con andamento appen- 
ninico, un'altra importante faglia con direzione N-S e a carattere paraforico è presente nella Valle del Melfa.

Interessante $\dot{e}$ notare che le faglie di tipo appenninico in relazione all'intera zona, M. Cairo, sono scarse e poco evidenti (1), contrariamente a quello che lo sviluppo delle isosiste avrebbe fatto pensare. Ia causa principale di tale propagazione rimane esclusivamente legata alla natura delle formazioni affioranti.

Tabella 5 - Valori del CoEfriciente di attenuazione def.jenergia MaCrosismica; TERremoto Delia Bassa Ciociaria.

\begin{tabular}{|c|c|c|}
\hline $\begin{array}{l}\text { Direzione dallepicentro } \\
\text { pereorso nelle formazioni }\end{array}$ & .1 & $\alpha \circ \bar{\alpha}$ \\
\hline $\begin{array}{l}\text { Nord-Ovest } \\
\text { _- lelle strutture dei Simbruini Er- } \\
\text { nici e copertura quaternaria }\end{array}$ & 27 & $0,0296 \pm 0,0016$ \\
\hline $\begin{array}{l}\text { Sucl-Est } \\
\text {-.. delle strutture dei Simbruini Er- } \\
\text { nici, coperture quaternarie }\end{array}$ & 13 & $0,0382 \pm 0,0104$ \\
\hline Norel & 7 & 0,0564 \\
\hline $\begin{array}{l}\text { - lelle strutture dei simbruini Er- } \\
\text { nici e della Marsiea sud-oceiden- } \\
\text { tale }\end{array}$ & $\begin{array}{l}9 \\
10\end{array}$ & $\begin{array}{l}0,0919 \\
0,1223\end{array}$ \\
\hline
\end{tabular}

$2.7-21$ MagGio

Cimento

\section{Dati epicentrali}

$H_{\mathrm{o}}=09^{\mathrm{h}} 03^{\mathrm{m}} 58^{\mathrm{s}} ; \varphi=40^{\circ} 19^{\prime} \mathrm{N} \lambda=14^{\circ} 58^{\prime} \mathrm{E} ; h=14 \mathrm{~km} ; M=3,7$ (RMP); $I_{\mathrm{o}}=\mathrm{V} \cdot \mathrm{VI}$

Altre determinazioni epicentrali

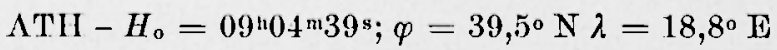

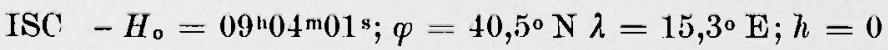




\section{Notizie macrosismiche}

Il Cilento in genere, e l'occidentale in particolare, presenta caratteristiche di asismicità. L'attuale evento è da considerarsi straordinario meritando una particolare attenzione.

Il sisma è stato sentito di:

V a Laureana, Agropoli, S. Maria di Castellabate, Castellabate, Montecorice, Torchiara, Rutino, Campora.

IV a Lustra, Prignano Cilento, Ogliastro Cilento, Perito, Serramezzana.

III a Stella Cilento, Cicerale, Giungano, Castel S. Lorenzo, Cannalunga, Ceraso.

II a Pisciotta, Montano Antilia, Laurino, Sala Consilina, S. Rufo, S. Pietro al Tunagro, S. Arsenio, Battipaglia.

Le isosiste presentano una regolare distribuzione del fenomeno macrosismico con debole prevalenza per la direzione E-O, fig. 9. In direzione NO si spingono oltre la formazione argilloso-calcarea della piana del Sele. Verso SE l'isosista di II si estingue in corrispondenza

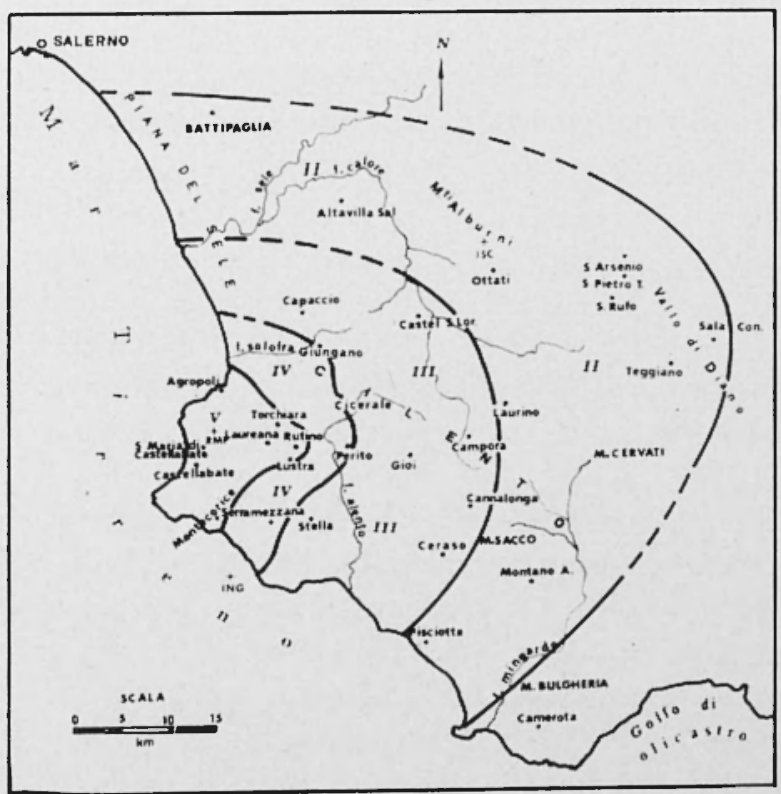

Fig. 9 - Isosiste del terremoto del Cilento, 21 Maggio 1969. 
del massiccio calcareo di MI. Bulgheria. Aleuni fenomeni di esaltazione dell'energia macrosismica si hanno in corrispondenza delle rastremazioni della formazione calcareo argillosa. I coefficienti di attenuazione dell'energia macrosismica in superficie sono riportati in tab. 6 .

\section{TABELLA 6}

\begin{tabular}{|c|c|c|}
\hline $\begin{array}{l}\text { Direzione dall'epicentro } \\
\text { pereorso nelle formazioni }\end{array}$ & 1 & $\alpha \circ \bar{a}$ \\
\hline $\begin{array}{l}\text { Nord-Nord Ovest } \\
-- \text { miste della serie Campano Lueana }\end{array}$ & 31 & $0,0496 \pm 0,0025$ \\
\hline $\begin{array}{l}\text { list } \\
\text { - eoceniche e del sopracretaceo del- } \\
\text { la serie Campano Lacana }\end{array}$ & 55 & $0,0288 \pm 0,0064$ \\
\hline $\begin{array}{l}\text { Sud-Est } \\
\text { - eoceniche fino al bordo della } \\
\text { struttura liassica di } \mathrm{N} \text {. Bulgheria }\end{array}$ & 42 & $0,0297 \pm 0,0005$ \\
\hline
\end{tabular}

Il 29 Marzo e il 2 Aprile, si sono verificati due eventi con ipocentro profondo a pochi chilometri dalle coste del Cilento. Tali eventi potrebbero essere in connessione con l'attuale sisma.

\section{Considerazioni tettoniche}

La zona epicentrale poggia sulle formazioni fliscioidi del complesso arenaceo-marnoso del Creta superiore, questa formazione non presenta in superficie, nella zona epicentrale, fagliamenti di importanza rilevante.

Il substrato è costituito da una struttura rigida di dolomie cristalline (Trias), interessate principalmente da dislocazioni tettoniche con andamento NO-SE. Questa struttura non pnò essere la sede dell'ipocentro trovandosi approssimativamente, nella zona epicentrale, ad una profonclità massima di $6000 \mathrm{~m}$.

Le incerte notizie sulla giacitura dello strato del granito, non permettono d'ipotizzare una qualsiasi giustificazione al movimento sismico. 


\section{8 - 2 Luglio MoN'TI DELLA TOLFA}

\section{Dati epicentrali}

$H_{\mathrm{o}}=07^{ }{ }^{\circ} 55^{\mathrm{m}} 43^{\mathrm{s}} ; \varphi=42^{\circ} 11^{\prime} \mathrm{N} \lambda=12^{\circ} 00^{\prime} \mathrm{E} ; h=8 \mathrm{~km} ; M=4,4$ (RMP); $I_{\mathrm{o}}=\mathrm{VII}$

\section{Altre determinazioni epicentrali}

USCGS $-H_{\mathrm{o}}=07^{\mathrm{n}} 55^{\mathrm{m}} 45^{\mathrm{s}} ; \varphi=42,3^{\circ} \mathrm{N} \lambda=12,1^{\circ} \mathrm{E} ; h=33 \mathrm{~km} ; M=4,4$ (CGS)

BCIS $-H_{\mathrm{o}}=07^{\mathrm{n}} 55^{\mathrm{m}} 45^{\mathrm{s}} ; \varphi=42,1^{\circ} \mathrm{N} \lambda=11,9^{\circ} \mathrm{E}$

ISC $-H_{0}=07^{\mathrm{n}} 55^{\mathrm{m}} 47^{\mathrm{s}} ; \varphi=42,1^{\circ} \mathrm{N} \lambda=11,5^{\circ} \mathrm{E} ; h=33 \mathrm{~km} ; M=4,4$

Per la prima replica

USCGS $-H_{0}=08^{\mathrm{n}} 02^{\mathrm{m}} 59^{\mathrm{s}} ; \varphi=42,3^{\circ} \mathrm{N} \lambda=12,2^{\circ} \mathrm{E} ; h=33 \mathrm{~km} ; M=4,5$

BCIS $-H_{0}=08^{11} 02^{\mathrm{m}} 53^{\mathrm{s}} ; \varphi=42,1^{\circ} \mathrm{N} \lambda=11,9^{\circ} \mathrm{E}$

ISC $-H_{0}=08^{n} 02^{m} 57 \mathrm{~s} ; \varphi=42,0^{\circ} N \lambda=11,8^{\circ} \mathrm{E} ; h=33 \mathrm{~km}$

\section{Notizie macrosismiche}

L'area tolfetana risultava storicamente asismica, era interessata solo marginalmente dagli eventi sismici dei centri circostanti come Bolsena, Monti Volsini, Monti Cimini.

Il periodo sismico della Tolfa, studiato da MI. De Panfilis ( $\left.{ }^{10}\right)$, è durato dal 2 al 9 Luglio e sono state registrate 15 scosse. Le principali di esse sono riportate in tabella 7 . Le coordinate epicentrali delle repliche data l'approssimazione ottenuta nel calcolo, possono considerarsi coincidenti con quelle della scossa principale.

Tabelata 7

\begin{tabular}{|c|c|c|c|c|}
\hline Data & $\begin{array}{c}\text { Ora } \\
\text { T.M.G. } \\
h \quad m\end{array}$ & $h$ & $M$ & $I_{\mathrm{o}}$ \\
\hline 2 I unglio & $\begin{array}{ll}08 & 03\end{array}$ & 11 & 4,4 & V.VI \\
\hline 2 Luglio & $\begin{array}{ll}08 & 08\end{array}$ & 9 & 3,5 & III-IV \\
\hline 2 Isuglio & $08 \quad 21$ & 8 & 3,5 & IV \\
\hline 2 Iuglio & 0945 & 8 & 2,8 & III \\
\hline 2 Iuglio & 1101 & 8 & 2,8 & II-III \\
\hline 2 Isuglio & 1344 & 8 & 2,8 & II-III \\
\hline
\end{tabular}


Sono state raccolte le seguenti notizie macrosismiche $\left({ }^{10}\right)$

VII a Civitella Cesi, Rota.

VI a Allumiere, Bagni di Stigliano, Barbarano Romano, Civitavecchia, Tolfa, Veiano.

V a Aurelia, Bassano Romano, Blera, Canale Monterano, Capranica, Castel Giuliano, Cura di Vetralla, Manziana, Monte Romano, Montevirginio, Oriolo Romano, S. Marinella, Sasso, Vetralla, Villa S. Giovanni in Tuscia.

IV-V a Ronciglione

IV a Bracciano, Caprarola, Cerveteri, Mazzano Romano, Montefiascone, Piansano, S. Martino al Cimino, S. Severa, Sutri, Viterbo.

III a Canepina, Carbognano, Ceri, Fabrica di Roma, Nonterosi, Tarquinia, Trevignano Romano, Tuscania, Vallerano, Vignanello, Vigna di Valle.

II a Anguillara Sabazia, Arlena di Castro, Bagnoregio, Calcate, Canino, Capodimonte, Castel S. Elia, Corchiano, Ladispoli, Latera, Marta, Montalto di Castro, Nepi, Onano, Palidoro, Palo, Pitigliano, S. Lorenzo Nuovo, Tessennano, Valentano, Vitorchiano.

Le isosiste (fig. 10) hanno un andamento molto regolare se si escludono alcune difformità dovute a condizioni geologiche locali. Esse fino al IVo presentano una debole preferenza per l'andamento antiappenninico, poi tendono ad attenuarsi fortemente in tale direzione e verso Roma. In direzione NO si ampliano oltre il lago di Bolsena. interessando i Monti Volsini e la Maremma meridionale. I valori del coefficiente di attenuazione sono riportati in tabella 8.

\section{Considerazioni geologiche}

La formazione più antica che si riscontra nella zona epicentrale ì il calcare massiccio costituente il " complesso basale " databile forse al Lias inf-medio (13). I sistemi di faglia che interessano il basamento hanno andamento NO-SE in modo predominante, NE-SO secondariamente, molto meno frequentemente compare la direzione meridiana. Lungo tali direttrici s'impostano le manifestazioni vulcaniche della regione. Secondo Iauro e Negretti (16) ed altri, le manifestazioni vulcaniche sono attribuibili ad un magma anatettico di natura sialica, 


\begin{tabular}{|c|c|c|}
\hline $\begin{array}{l}\text { Direzione dall'epicentro } \\
\text { percorso nelle formazioni }\end{array}$ & $\Delta$ & $\begin{array}{lll}\alpha & 0 & \alpha\end{array}$ \\
\hline Nord & 34 & $0,0354 \pm 0,0025$ \\
\hline $\begin{array}{l}\text { - sedimentaric co-plioceniche con } \\
\text { copertura piroclastica prevalente }\end{array}$ & & \\
\hline Nordi-Est & 13 & 0,0179 \\
\hline - dell'apparato Vuleanico Vicano & $\begin{array}{l}25 \\
28\end{array}$ & $\begin{array}{c}0,0303 \pm 0,0025 \\
0,0+13\end{array}$ \\
\hline $\begin{array}{c}\text { Sud-ovest } \\
\text { - sedimentarie eo-plioceniche }\end{array}$ & 24 & $0,018 \pm 0,002$ \\
\hline Sud-Sud Est & 20 & $0,0247 \pm 0,0013$ \\
\hline $\begin{array}{l}\text { - sedimentarie eo-plioceniche con } \\
\text { debole copertura piroclastica }\end{array}$ & 24 & 0,0336 \\
\hline
\end{tabular}

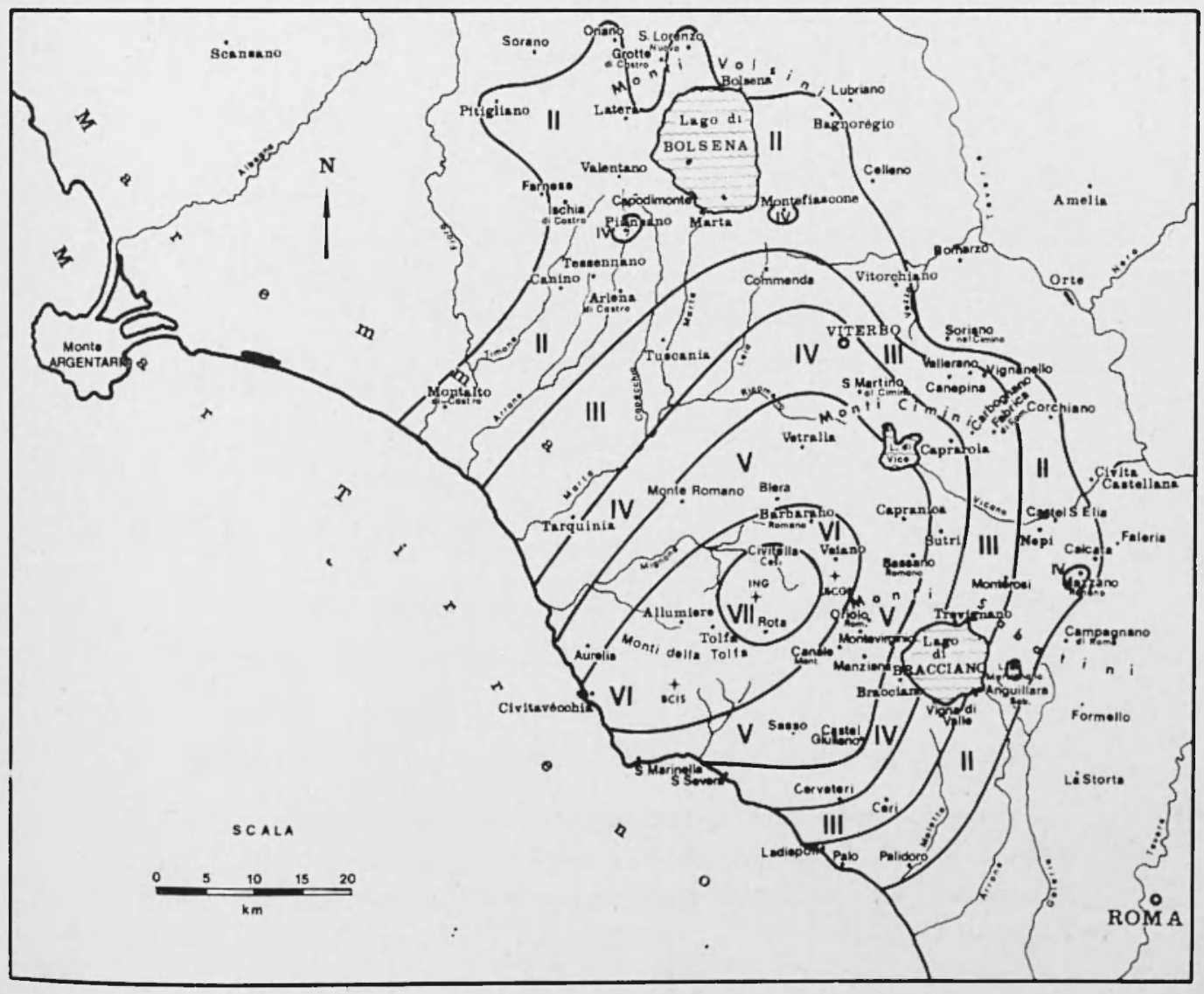

Fig. 10 - Isosiste del terremoto della Tolfa, 2 Luglio 1969 (da De Panfilis). 
con accentuate differenziazioni, la cui ascesa andrebbe attribuita alla fase post-orogenica del sollevamento appenninico.

L'attuale movimento sismico ha interessato presumibilmente la direttrice tettonica antiappenninica, Tolfa-Lago di Vico. Lungo tale direttrice si riscontrano storicamente diversi centri sismici attivi, i cui eventi in genere, sono dovuti a fenomeni di assestamento tettonico locale.

Casentino

Dati epicentrali

$H_{\mathrm{o}}=09^{\mathrm{h} 20^{\mathrm{m}} 57^{\mathrm{s}}} ; \varphi=43^{\circ} 42^{\prime} \mathrm{N} \lambda=11^{\circ} 56^{\prime} \mathrm{E} ; h=10 \mathrm{~km} ; M=4,1$ (RMP); $I_{\mathrm{o}}=\mathrm{VI}$

Altre determinazioni epicentrali

USCGS $-H_{\mathrm{o}}=09^{\mathrm{h}} 21^{\mathrm{m}} 07^{\mathrm{s}} ; \varphi=44,2^{\circ} \mathrm{N} \lambda=11,9^{\circ} \mathrm{E} ; h=33 \mathrm{~km} ; M=4,1$ (CGS)

BCIS $-H_{\mathrm{o}}=09^{\mathrm{h}} 21^{\mathrm{m}} 03^{\mathrm{s}} ; \varphi=44,0^{\circ} \mathrm{N} \lambda=12,0^{\circ} \mathrm{E}$

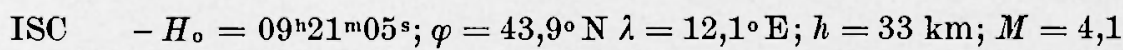

Per la replica del 10-VIII

USCGS $-H_{\mathrm{o}}=21^{\mathrm{n} 16^{\mathrm{m}} 24^{\mathrm{s}}} ; \varphi=44,0^{\circ} \mathrm{N} \lambda=12,1^{\circ} \mathrm{E} ; h=33 ; M=4,0$ (CGS)

BCIS $-H_{\mathrm{o}}=21^{\mathrm{n}} 16^{\mathrm{m} 20^{\mathrm{s}}} ; \varphi=44,0^{\circ} \mathrm{N} \lambda=12,0^{\circ} \mathrm{E}$

ISC $-H_{\mathrm{o}}=21^{\mathrm{h}} 16^{\mathrm{m} 27^{\mathrm{s}}} ; \varphi=43,9^{\circ} \mathrm{N} \lambda=12,2^{\circ} \mathrm{E} ; h=60 \pm 31$

\section{Notizie macrosismiche}

Questo terremoto si ̀̀ manifestato durante un breve periodo sismico tab. 9, la scossa principale è stata preceduta da una leggera premonitrice e seguita da tre repliche. L'epicentro cade tra M. Penna e l'Alpe di Catenaia. 
Tabella 9

\begin{tabular}{|c|c|c|c|c|c|c|}
\hline Data & $\begin{array}{c}\text { Ora } \\
\text { T.M.G. } \\
h \quad m\end{array}$ & $\begin{array}{c}\text { Coord. } \\
\varphi\end{array}$ & $\underset{\lambda}{\text { Epic. }}$ & $h$ & $\begin{array}{c}I I \\
(\mathrm{RMP})\end{array}$ & $I_{0}$ \\
\hline 9 Agosto & $09 \quad 11$ & $43^{\circ} 42^{\prime} \mathrm{N}$ & $11^{\circ} 56^{\prime} \mathrm{E}$ & 10 & 3,1 & II I \\
\hline 9 Agosto & 0923 & $43^{\circ} 42^{\prime} \mathrm{N}$ & $11^{\circ} 56^{\prime} \mathrm{E}$ & 10 & 3,3 & IV \\
\hline 10 Agosto & $21 \quad 16$ & $43^{\circ} 42^{\prime} \mathrm{N}$ & $11^{\circ} 56^{\prime} \mathrm{E}$ & 11 & 3,5 & IV $-\mathrm{V}$ \\
\hline 10 Agosto & 2124 & $43^{\circ} 42^{\prime} \mathrm{N}$ & $11^{\circ} 56^{\prime} \mathrm{E}$ & 9 & 3,0 & III \\
\hline
\end{tabular}

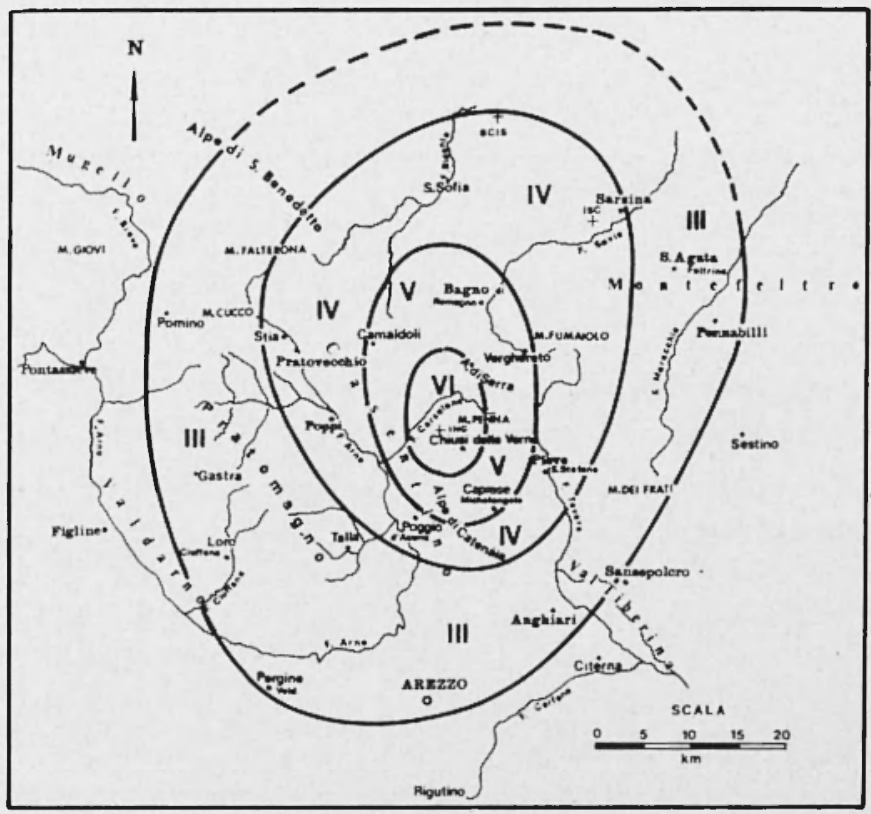

Fig. 11 - Isosiste del terremoto del Casentino, 9 Agosto 1969.

Le notizie macrosismiche raccolte per la scossa principale sono le seguenti:

VI a Chiusi della Verna.

V a Caprese Michelangelo, Bagno di Romagna, Verghereto, Camaldoli. 
IV a Pratovecchio, S. Sofia, Sarsina, Stia, Pieve S. Stefano, Poggio d'Acona.

IlI a S. Agata Feltrina, Pennabilli, Anghiari, Arezzo, Pergine Val d'Arno, Talla, Loro Ciuffena, Gastra, Pomino.

Le isosiste, fig. 11, hanno un andamento ellittico con una maggiore propagazione verso Nord. Gli effetti del terremoto non si sono sentiti nel Valdarno superiore, nella Val di Chiana, nella media Val Tiberina e lungo le pendici dell'Appennino Romagnolo, come pure nel Mugello e nell'Alpe di S. Benedetto. L'isosisma di III ha invece oltrepassato la dorsale del Pratomagno-Alpe di Poti, ed il Valdarno inferiore. In tabella 10 sono riportati i valori del coefficiente di attenuazione dell'energia macrosismica.

TABELLA 10

\begin{tabular}{|c|c|c|}
\hline $\begin{array}{c}\text { Direzione dallepicentro } \\
\text { percorso nelle formazioni }\end{array}$ & $\Delta$ & $\alpha$ o $\bar{\alpha}$ \\
\hline $\begin{array}{c}\text { Nord-Ovest } \\
\text { - eoceniche, parallelamente all'asse } \\
\text { di allineamento dei bacini lacu- }\end{array}$ & 24 & 0,0439 \\
stri pliocenici & 10 & 0,0253 \\
Nord-Est & 32 & $0,0199 \pm 0,0027$ \\
$\begin{array}{l}\text { eoceniche, perpendicolarmente al- } \\
\text { penninico }\end{array}$ & \\
Sud-Ovest & 24 & $0,0458 \pm 0,0030$ \\
\hline $\begin{array}{l}\text { eoceniche e plioceniche alluvio- } \\
\text { nali dell Alta Val Tiberina }\end{array}$ &
\end{tabular}

\section{Considerazioni geologiche}

Le caratteristiche tettoniche della regione sono date da una serie di pieghe sinclinali e anticlinali con vergenza a $\mathrm{NE}$ e dall'immersione della "serie toscana" sotto i complessi Tosco-Emiliani. I terreni compresi nell'area megasismica sono quelli della serie toscana che hanno uno spessore, in questa zona, di $5200 \mathrm{~m}$ circa. L'ipocentro si troverebbe cosi compreso tra il tetto dello strato granitico ed il complesso basale sedimentario. 


\subsection{0 - 11 Agosto Lago Trasimeno}

Dati epicentrali

$H_{\mathrm{o}}=13^{\mathrm{n}} \tilde{5} \tilde{5}^{\mathrm{m}} 08^{\mathrm{s}} ; \varphi=43^{\circ} 02^{\prime} \mathrm{N} \lambda=12^{\circ} 12^{\prime} \mathrm{E} ; h=17 \mathrm{~km} ; M=4, \tilde{5}$ (RMP);

$I_{\mathrm{o}}=\mathrm{VII}$

Altre determinazioni epicentrali

USCGS $-H_{0}=13^{\mathrm{n}} 55^{\mathrm{m}} 12^{\mathrm{s}} ; \varphi=43,2^{\circ} \quad \mathrm{N} \lambda=12,4^{\circ} \mathrm{E} ; h=33 \mathrm{~km} ;$ $M=4,6$ (CGS)

BCIS $-H_{\mathrm{o}}=13^{\mathrm{h}} 55^{\mathrm{m}} 09^{\mathrm{s}} ; \varphi=43,2^{\circ} \quad \mathrm{N} \lambda=12,2^{\circ} \quad \mathrm{E}$

ISC $-H_{0}=13^{\mathrm{n}} 55^{\mathrm{m}} 09^{\mathrm{s}} ; \varphi=43,11^{\circ} \mathrm{N} \lambda=12,21^{\circ} \mathrm{E} ; h=2 \pm 7,1 \mathrm{~km}$; $M=4,1$

\section{Notizie macrosismiche}

Il terremoto è stato sentito di:

VII a Mugnano, Fontignano, S. Martino dei Colli, Castiglione della Valle,

VI-VII a Panicale, Tavernelle,

VI a Migliano, Marsciano, Spina, Morcella, Magione,

V a Città della Pieve, Deruta, Perugia, Lisciano, Terontola, Cortona, Cetona,

IV a Sarteano, S. Casciano dei Bagni, Bastia, Umbertide, Montone, Morra, Castiglion Fiorentino, Marciano, Sinalunga, Montepulciano

III a Pergine Val d'Arno, Monte S. Savino, S. Quirico d'Orcia, Radicofani, Abbadia S. Salvatore, Orvieto, Todi, Valfabbrica, S. Martino in Colle, Città di Castello, Arezzo,

II a Loro Ciuffena, Terranova Bracciolini, Anghiari, Gubbio, Foligno.

L'area di transizione costituita dal passaggio tra serie umbromarchigiana e toscana, nella zona del Lago Trasimeno è stata interessata nel volgere di 9 giorni da diversi eventi sismici distribuiti in un raggio di $25 \mathrm{~km}$ dall'epicentro attuale. Il maggiore di tali eventi è quello di Mngnano, al quale alle $15^{\mathrm{h}} 40^{\mathrm{m}} 25^{\mathrm{s}}$ è seguita una replica di III-IV grado. 
La scossa, molto intensa, ha provocato gravi danni alle costruzioni in diversi comuni dell'area epicentrale con circa 200 case lesionate di cui 50 inabitabili. Te isosiste, fig. 12 presentano un'uniforme propagazione dell'energia macrosismica nelle varie direzioni, una debole preferenza si nota in direzione $\mathrm{N}$-NO. In tabella 11 sono riportati i valori del coefficiente di attenuazione dell'energia macrosismica.

Tabelia 11

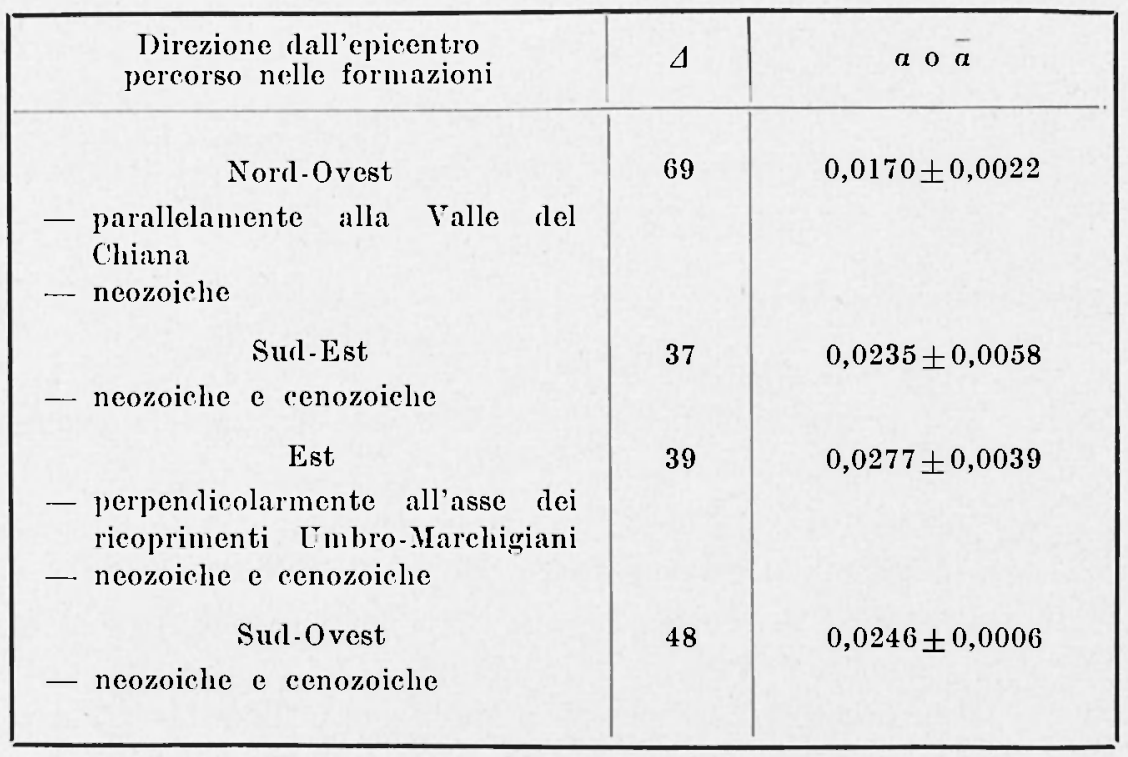

\section{Considerazioni geologiche}

L'area interessata dal sisma è costituita dagli accavallamenti dovuti al diverso evolversi, a partire dal giura inferiore, della serie toscana e umbro-marchigiana. Le dislocazioni tettoniche più evidenti sono quelle della grande faglia sepolta lungo il bordo sud-orientale della Val di Chiana. Trasversalmente a questa, l'altra grande dislocazione che procede dal M. Amiata fino a chiudere la depressione sud del lago Trasimeno. Si può affermare che lungo le linee strutturali impostate in epoche precedenti continuano i movimenti tettonici; ne testimoniano i sismi con ipocentri prossimi alla prima discontinuità della crosta, come questo, i depositi attuali di travertino e l'estesa distribuzione di sorgenti termominerali. 


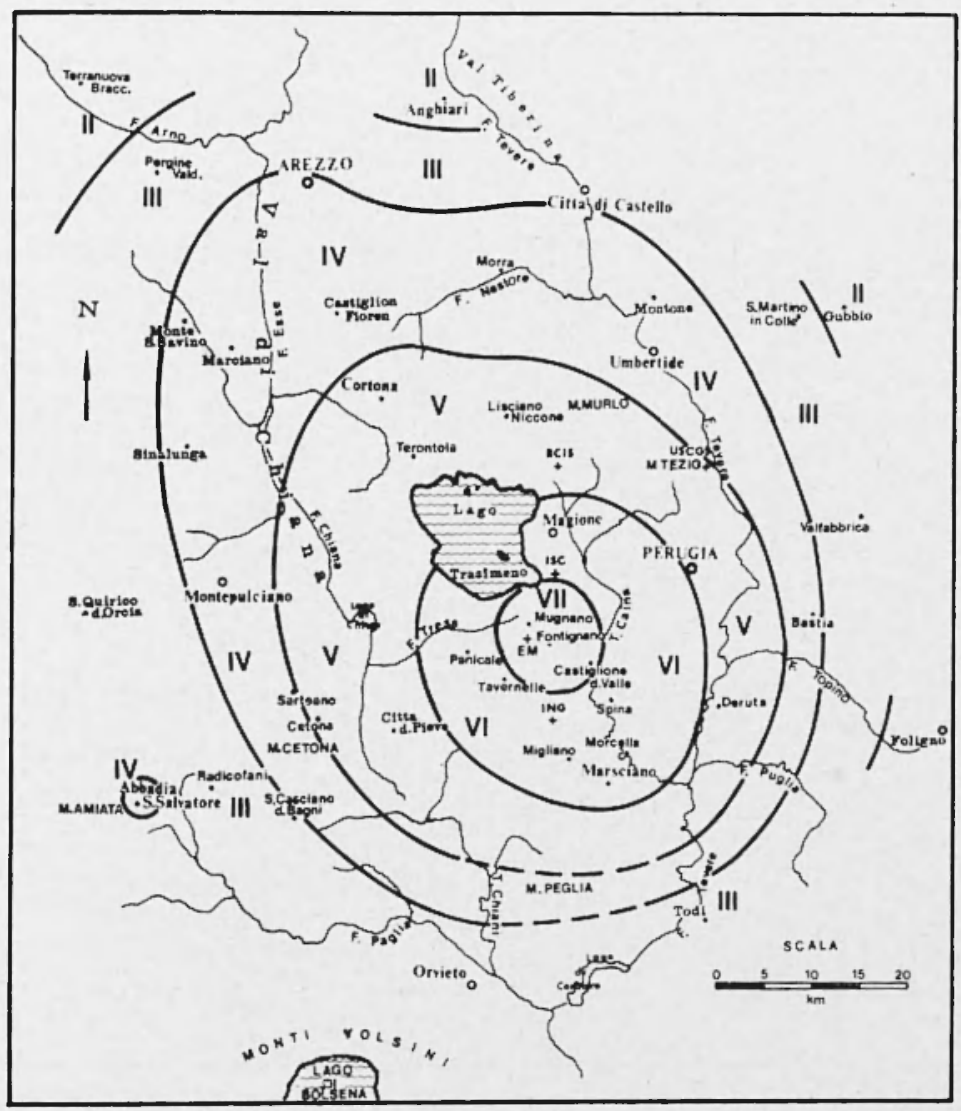

Fig. 12 - Isosiste del terremoto del Iaago Trasimeno, 11 Agosto 1969.
2.11 - 26 SetTembRE
Gran Sasso D'Italia

\section{Dati epicentrali}

$H_{\mathrm{o}}=23^{\mathrm{h}} 40^{\mathrm{m}} 39^{\mathrm{s}} ; \varphi=42^{\circ} 33^{\prime} \mathrm{N} \lambda=13^{\circ} 36^{\prime} \mathrm{E} ; h=24 \mathrm{~km} ; M=4,5(\mathrm{RMP}) ;$

$I_{0}=\mathrm{VI}$

\section{Notizie macrosismiche}

L'evento è stato preceduto da una scossa di III-IV alle $23^{\mathrm{h}} 40^{\mathrm{m} 25^{\mathrm{s}}}$. 


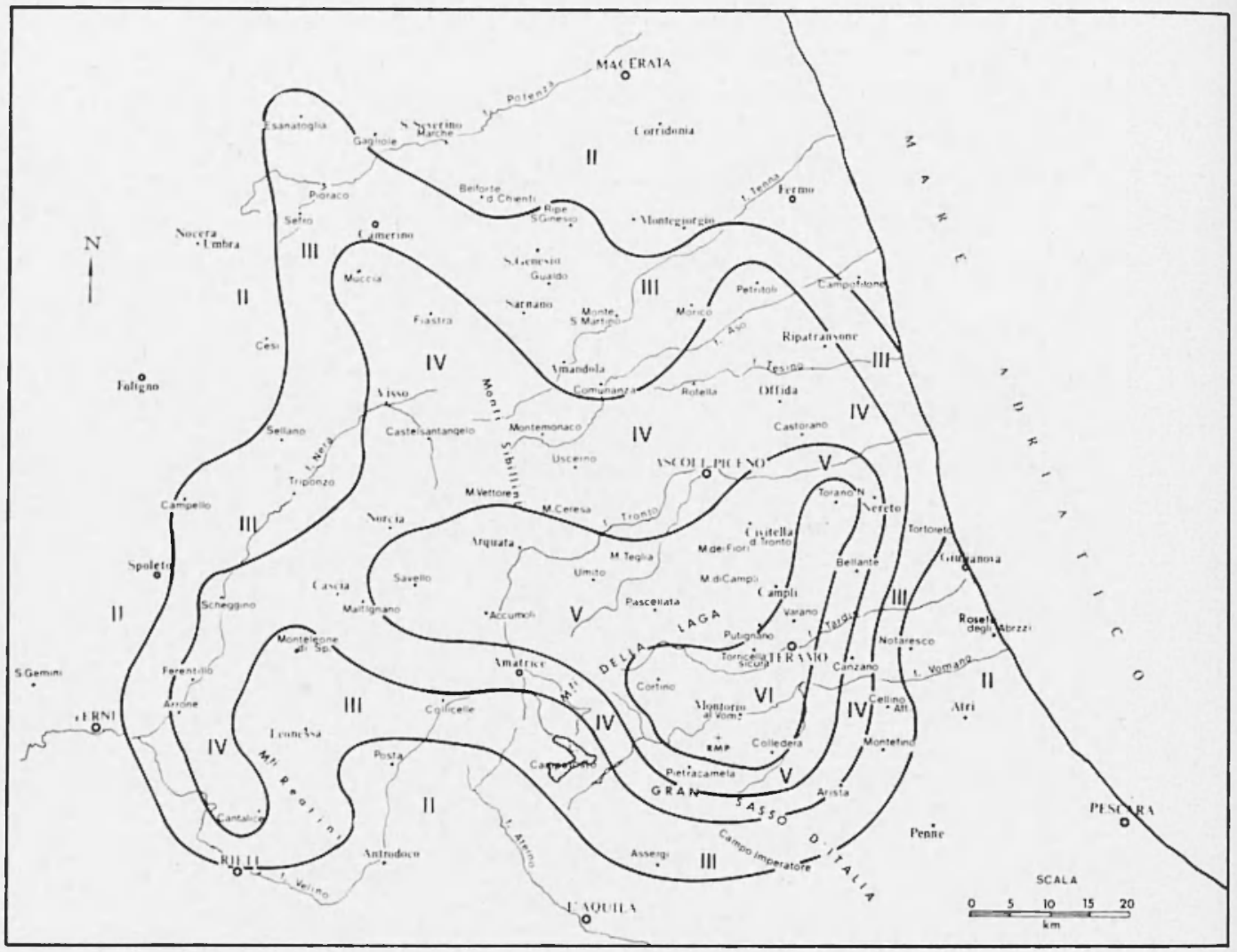

Figr. 13 - Isosiste del terremoto del Gran Sasso d'Italia, 26 Settembre 1969.

Sono state laccolte le seguenti notizie macrosismiche:

VI a Montorio al Vomano, Cortino, Colledera, Varano, Torano Nuovo, Torricella Sicura.

V a Campli, Putignano, Civitella del Tronto, Umito, Accumoli, Arquata, Pietracamela, Bellante, Nereto.

IV a Castorano, Canzano, Maltignano, Cantalice, Ferentillo, Scheggino, Norcia, Castel S. Angelo, Visso, Muccia, Fiastra, Montemonaco, Petritoli.

III a Esanatolia, Pioraco, Camerino, Gualdo, Ripe S. Genesio, Monte S. Martino, Tortoreto, Cellino Attanasio, Montefino, Assergi, Monteleone di Spoleto, Leonessa, Campello sul Clitumno, Sellano, Sefro. 
II a Foligno, Spoleto, S. Gemini, Terni, Posta, Antrodoco, Penne, Atri, Notaresco, Giulianova, Gagliole, S. Severino Marche, Belforte del Chienti, Montegiorgio, Campofilone.

Nel comune di Torano Nuovo si sono formati dei Vulcanelli di fango con emissione di acque e gas solforosi.

L'area epicentrale interessa in massima parte i terreni della serie di "Transizione " e la copertura arenaceo-argillosa neogenica.

Le isosiste, fig. 13, assumono un andamento alquanto contorto, causato prevalentemente dall'influsso che la geologia di superficie ha nella propagazione degli effetti macrosismici.

In linea di massima si riscontra, una fortissima attenuazione in corrispondenza del Gran Sasso d'Italia dovuta prevalentemente alla

\section{TABELLA 12}

\begin{tabular}{|c|c|c|}
\hline $\begin{array}{l}\text { Direzione dall epicentro } \\
\text { percorso nelle formazioni }\end{array}$ & $\therefore$ & $\alpha \circ \bar{\alpha}$ \\
\hline $\begin{array}{l}\text { Nord } \\
\text { - coceniche e plioceniche della fos- } \\
\text { sa marehigiana, e lungo il bordo } \\
\text { della gessoso-solffera }\end{array}$ & 61 & $0,0149 \pm 0,0007$ \\
\hline $\begin{array}{l}\text { Sud } \\
\text { coceniche e della facies di transi- } \\
\text { zione della serie laziale-abruz- } \\
\text { zese }\end{array}$ & 18 & $0,1413 \pm 0,0182$ \\
\hline $\begin{array}{l}\text { Nord-Ovest } \\
\text { - coceniche e della gessoso-solfifera } \\
\text { della fossa marchigiana e preva- } \\
\text { lentemente in quella della facies } \\
\text { Umbro-Narehigiana }\end{array}$ & 97 & $0,0081 \pm 0,0006$ \\
\hline $\begin{array}{l}\text { Ovest-Nord Ovest } \\
\text { - perpendicolarmente all asse della } \\
\text { virgazione Sabina } \\
\text { - coceniche e della gessoso-solfifera } \\
\text { della fossa marchigiana e in parte } \\
\text { in quella della facies Umbro- } \\
\text { Marchigiana }\end{array}$ & 74 & $0,0118 \pm 0,002$ \\
\hline $\begin{array}{c}\text { Norl-Est } \\
\text { - della fossa marchigiana }\end{array}$ & 26 & $0,0472 \pm 0,0091$ \\
\hline
\end{tabular}


notevole fagliatura delle formazioni calcaree mesozoiche. Ugualmente si sono comportati i calcari giurassici di M. dei Fiori, (in coincidenza con l'anticlinale Ascolana), una discreta attenuazione si registra anche in coincidenza con la copertura quaternaria in prossimità della costa.

Delle deboli attenuazioni si riscontrano in direzione dei Monti Sibillini e verso Nord lungo i terreni pliocenici. Il massimo di propagazione si ha lungo tutta la fascia di virgazione appenninica tra Fabriano e Rieti. I valori relativi al coefficiente di attenuazione dell'energia macrosismica sono riportati in tab. 12.

\section{Lineamenti tettonici}

L'epicentro cade nel passaggio tra la facies Umbro-Marchigiana e Laziale-Abruzzese. La facies Umbro-Marchigiana ha una struttura che nel complesso presenta un comportamento prevalentemente plastico. Il basamento calcareo-dolomitico invece ha in relazione alle sollecitazioni meccaniche un carattere rigido. Il complesso ha uno stile tettonico a pieghe con grandi anticlinali generalmente troncate da faglie dirette. Sono anche presenti pieghe-faglie e pieghe-rovesciate. La facies Laziale-Abruzzese ha una struttura totalmente rigida, con grossi blocchi monoclinali delimitati e svincolati gli uni rispetto agli altri da faglie con direzione E-O, N-S, NO-SE, NE-SO.

Le strutture presentano una vergenza prevalentemente Adriatica.

\section{$2.12-9$ OTTOBRE}

VALLE DI SUSA

Dati epicentrali

$H_{\mathrm{o}}=03^{\mathrm{n}} 31^{\mathrm{m}} 35^{\mathrm{s}} ; \varphi=45^{\circ} 05^{\prime} \mathrm{N} \lambda=7 \circ 22^{\prime} \mathrm{E} ; h=13 \mathrm{~km} ; M=4,2$ (CGS); $I_{0}=\mathrm{VI}$

\section{Altre determinazioni epicentrali}

USCGS $-H_{\mathrm{o}}=03^{\mathrm{h}} 31^{\mathrm{m}} 35^{\mathrm{s}} ; \varphi=45,0^{\circ} \mathrm{N} \lambda=7,7^{\circ} \mathrm{E} ; h=33 \mathrm{~km} ; M=4,2$

BCIS $-H_{\mathrm{o}}=03^{\mathrm{n}} 31^{\mathrm{m}} 35^{\mathrm{s}} ; \varphi=45,07^{\circ} \mathrm{N} \lambda=7,35^{\circ} \mathrm{E}$

ISC $\quad-H_{\mathrm{o}}=03^{\mathrm{n}} 31^{\mathrm{m}} 36^{\mathrm{s}} ; \varphi=44,95^{\circ} \mathrm{N} \lambda=7,4^{\circ} \mathrm{E} ; h=33 \mathrm{~km}$

\section{Notizie macrosismiche}

Il sisma si è sviluppato e propagato in corrispondenza della sinclinale che costituisce la Valle di Susa. 
Sono state raccolte le seguenti notizie macrosismiche:

VI a Avigliana, Rudiana, Rosta

V a Villar, Vaie, Coazze, Giaveno, Rivoli, Piossasco, Grugliasco, Torino, Pianezza

IV a Cumiana, Orbassano, Rivolta di Torino, Nichelino, La Cassa, Chiamonte, Venaus, Susa, MIompantero, Gravero

III a Bardonecchia, Cesana Torinese, La Loggia

II a Poirino, Leini, Lombardone.

L'area epicentrale interessa prevalentemente i terreni quaternari costituiti dalle alluvioni recenti e dalle morene di ghiacciai wurmiani.

Ise isosiste, fig. 14, presentano un andamento parallelo alla Valle di Susa con una maggiore propagazione verso Ovest, in corrispondenza

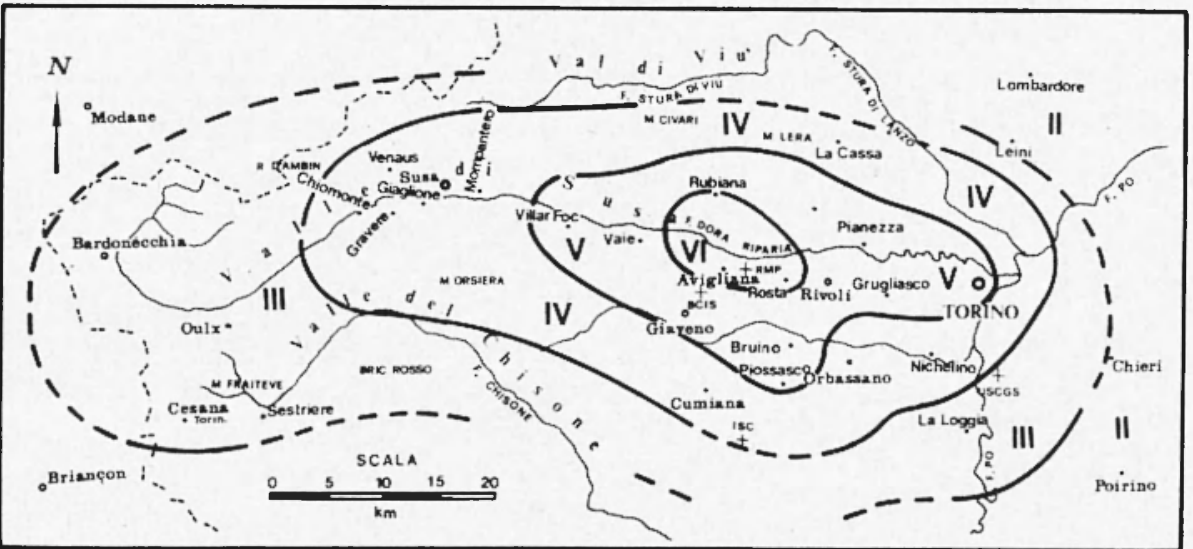

Fig. 14 - Isosiste del terremoto della Val di Susa, 9 Ottobre 1969.

della serie dei Monti di Ambin. In direzione opposta si ha una forte attenuazione al bordo del bacino terziario piemontese. A Nord nella zona costituita dalle "pietre verdi " l'isosisma di IV non oltrepassa la Valle di Viù. A sud sono stati interessati anche i terreni della serie del massiccio Dora-Val Maira. L'ipocentro cade presumibilmente all'interno dello strato del granito. 


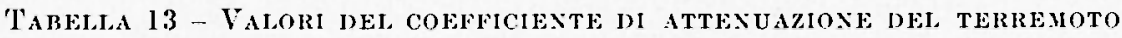
DELIA VAILLE DI SUSA.

\begin{tabular}{|c|c|c|}
\hline $\begin{array}{l}\text { Direzione dall'epicentro } \\
\text { percorso nelle formazioni }\end{array}$ & $\Lambda$ & $a \circ \bar{a}$ \\
\hline $\begin{array}{l}\text { Ovest } \\
\text { - perpendicolarmente ai rieopri- } \\
\text { menti penniniei } \\
\text { - costituite da rocee metamorfiche } \\
\text { in generale, calcescisti in parti- } \\
\text { colare } \\
\text { Est } \\
\text { - del bacino terziario piemontese } \\
\text { Sud } \\
\text { - parallelamente ai ricoprimenti } \\
\text { penninici } \\
\text { - di contatto tra sedimenti del ba- } \\
\text { cino piemontese e formazioni me- } \\
\text { tamorfiche }\end{array}$ & $\begin{array}{r}22 \\
27 \\
8 \\
15\end{array}$ & $\begin{array}{l}0,01447 \\
0,02386 \\
0,08024 \\
0,05909\end{array}$ \\
\hline
\end{tabular}

$2.13-14$ Novemiske

APPENINO IUUCANO

Dati epicentrali

$H_{0}=06^{n} 48^{\mathrm{m} 07^{\mathrm{s}}} ; q=40^{\circ} 35^{\prime} \mathrm{N} \lambda=15^{\circ} 34^{\prime} \mathrm{E} ; h=40 \mathrm{~km} ; M=4,1(\mathrm{RMP}) ;$ $I_{0}=\mathrm{V}$

Altre determinazioni epicentrali

USCGS $-06^{n}+8^{\mathrm{m}} 04^{\mathrm{s}} ; \varphi=40,7^{\circ} \mathrm{N} \lambda=15,7^{\circ} \mathrm{E} ; h=15 ; M=4, \tau$ (CGS)

ISO $\quad-06^{\mathrm{n}} 48^{\mathrm{m}} 0 \mathrm{7}^{\mathrm{s}} ; \varphi=40,7^{\circ} \mathrm{N} \lambda=15,7^{\circ} \mathrm{E} ; h=41 \pm \tau$

Notizie macrosismiche

Sono state raccolte le seguenti notizie macrosismiche:

V a Auletta, Pertosa, Satriano di Lucania, Picerno, Vietri di Potenza 
IV a Tito, Ruoti, Bella, Buccino, S. Angelo le Fratte

III a Castelgrancle, Potenza, Sala Consilina, S. Arsenio, Scorzo

II a Capo Sele, Castel Baronia, Castelfranci, Atriplada, Solofra, Tramutola, Montemuro.

Le isosiste, riportate in fig. 15, assumono un andamento molto irregolare ricalcando le principali strutture morfologiche della regione. L'area megasismica si sviluppa in direzione E-O interessando le for-

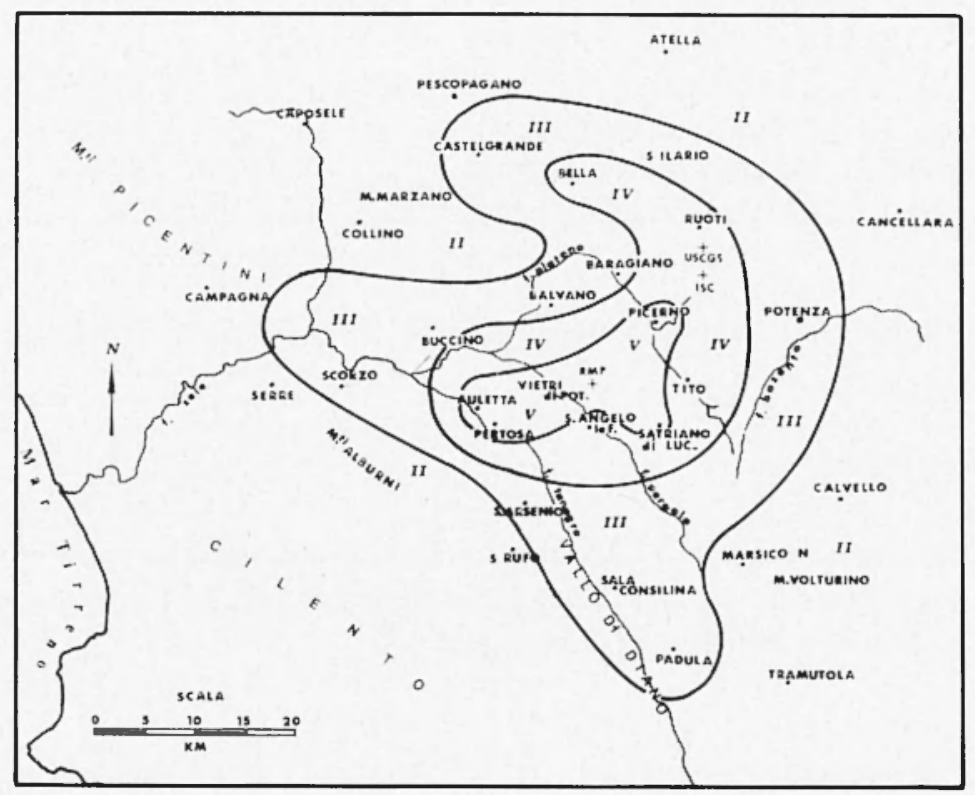

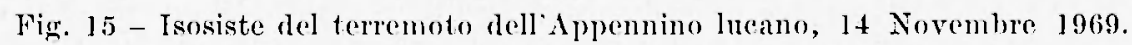

mazioni triassiche della serie calcareo-silico-marnosa. Questa seria presenta nella sua struttura una tendenza al rovesciamento verso oriente, creando cosi dei complessi con andamento centrale NO-SE, disposta ad arco con convessità ad Est. L'isosista di III, verso NO assume un andamento avvolgente in corrispondenza del massiccio calcareo di Monte Marzano mentre in direzione opposta s'inoltra nel Graben del Vallo di Diano. Le localitì settentrionali in cui il sisma i stato sentito di II si trovano nella Valle del Sele e Iungo il piede dei M. Picentini, a meridione queste localita giacciono in corrispondenza dell'alta 
Valle dell'Agri. I valori del coefficiente di attenuazione dell'energia macrosismica in superficie sono riportati in tabella 14 .

TABELLA 14

\begin{tabular}{|c|c|c|}
\hline $\begin{array}{l}\text { Direzione dallepicentro } \\
\text { percorso nelle formazioni }\end{array}$ & $\Delta$ & $\alpha \circ \bar{\alpha}$ \\
\hline $\begin{array}{r}\text { Ovest-Sud Ovest } \\
\text { - verso i Monti Alburni } \\
\text { - eoceniche e cretaciche }\end{array}$ & 16 & $0,1362 \pm 0,0329$ \\
\hline Sur-Sud Est & 10 & 0,18122 \\
\hline $\begin{array}{l}\text { - costituenti l'arco lucano, eoce- } \\
\text { niche e cretaciche in prevalenza }\end{array}$ & 34 & 0,04048 \\
\hline
\end{tabular}

L'ipocentro si troverebbe al disotto della Moho, nell'astenosfera.

\section{3 - Compiementi}

Altri eventi sismici - In quest'ultima parte del lavoro sono stati riportati i terremoti macrosismicamente sentiti in Italia, ma causati da eventi con epicentro in altri paesi.

\section{$3.1-3$ Aprile Telepena (Albania)}

Dati epicentrali

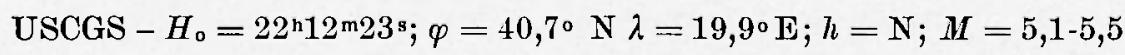
(CGS)

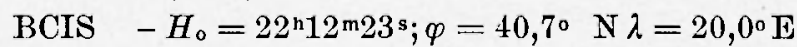

$\Delta$ TII $-H_{\mathrm{o}}=22^{\mathrm{h}} 12^{\mathrm{m}} 20^{\mathrm{s}} ; \varphi=40,5^{\circ} \mathrm{N} \lambda=19,9^{\circ} \mathrm{E} ; M=5,5$

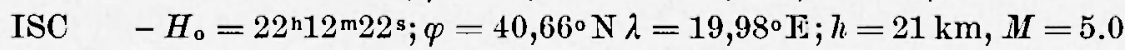

Il sisma il cui epicentro cade nella regione di Mallakastra è stato di VII MMI. Esso ha causato 2 morti, 126 feriti e notevoli danni. Un migliaio di case fortemente danneggiate o distrutte ed altre 2000 lie- 


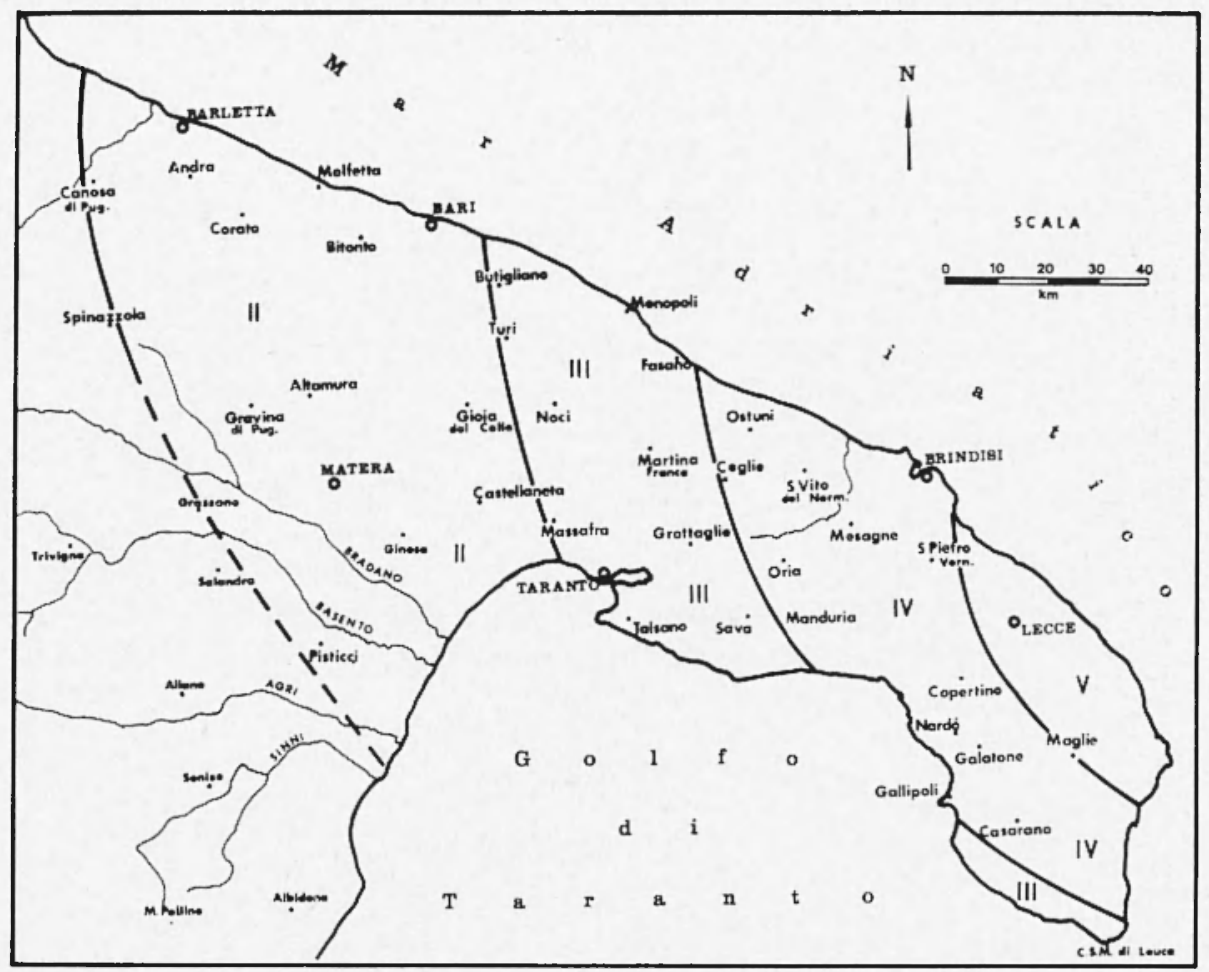

Fig. 16 - Isosiste, per la parte italiana, del terremoto di Telepena (Albania), 3 Aprile 1969.

vemente danneggiate. In Italia è stato sentito in provincia di Lecce, Bari, Brindisi, Taranto, in fig. 16 sono riportate le isosiste per la parte italiana. In provincia di Bari e Brindisi, nello stesso giorno, sono state sentite alcune repliche di grado tra il II ed il IV.

\section{2 - $1^{\circ}$ Givgno Postumia (Jugoslavia)}

\section{Dati epicentrali}

ISC $-H_{0}=07^{\mathrm{n}} 38^{\mathrm{m}} 38^{\mathrm{s}} ; \varphi=45,83^{\circ} \mathrm{N} \lambda=14,17^{\circ} \mathrm{E} ; h=45 \mathrm{~km}$

BCIS $-H_{0}=07^{\mathrm{n}} 38^{\mathrm{m}} 38^{\mathrm{s}} ; \varphi=45,8^{\circ} \quad \mathrm{N} \lambda=14,1^{\circ} \quad \mathrm{E}$ 
Il terremoto è stato valutato di IV grado all'epicentro. In Italia è stato sentito di II-III a Trieste e in alcuni paesi del Friuli.

\section{$3.3-26$ OT'TOBRE BANJA-LUKA (JUGOSLAVIA)}

Dati epicentrali

USCGS $-H_{\mathrm{o}}=15^{\mathrm{h} 36^{\mathrm{m}} 52 \mathrm{~s}} ; p=44,9^{\circ} \quad \mathrm{N} \lambda=17,3^{\circ} \mathrm{E} ; h=33 ; M=5,3$ (CGS)

BCIS $-H_{0}=15^{\mathrm{h}} 36^{\mathrm{m}} 5^{\mathrm{s}} \mathrm{s} ; p=44,9^{\circ} \mathrm{N} \lambda=17,3^{\circ} \mathrm{E}$

BEO - Epic. Macr. $\quad p=44^{\circ} 57^{\prime} \mathrm{N} \lambda=17^{\circ} 05^{\prime} \mathrm{E} ; M=5,6$

Il sisma è stato valutato di VII-VIII all'epicentro. Si sono avuti 15 morti e 200 feriti oltre a gravissimi danni alle costruzioni.

In Italia è stato sentito in tutto il Friuli (III grado) inoltre di $\mathrm{V}$ a Muggia e IV a Trieste.

3.4 - 27 OTTORRE BANJA-LUKA (JUGOSLAVIA)

Dati epicentrali

USCGS $-I_{0}=08^{\mathrm{h} 10 \mathrm{~m}} 58^{\mathrm{s}} ; \phi=44,9 \circ \mathrm{N} \lambda=17,2 \circ \mathrm{E} ; h=\mathrm{N} ; M=5,3$ BCIS $-H_{0}=08^{n} 10^{\mathrm{m}} 56^{\mathrm{s}} ; \phi=44,8^{\circ} \mathrm{N} \lambda=17,2^{\circ} \mathrm{E}$

Il terremoto si è manifestato d'intensiti compresa tra VIII-IX. Si sono avuti oltre 14 morti e 1100 feriti, 75.000 senza tetto a BanjaJuka.

Il $60 \%$ delle abitazioni distrutte irreparabilmente e tre villaggi vicini all'epicentro rasi al suolo.

In Italia è stato sentito di V nel Frinli e di II-IV grado nelle Marche e lungo la costa Arriatica. Nel triestino è stata anche avvertita la premonitrice delle $02^{\mathrm{h}} 55^{\mathrm{m}}$ di III-IV grado.

4. - JA SINMICITÀ DELLE ALPI MARITTIME E DELLE AREE ADIACENTI

Da una nota di M. Bossolasco e C. Eva (') abbiamo estratto i dati della Tab. 15 che riporta gli eventi sismici avvenuti nelle $\mathrm{Al}_{\text {pi }}$ Iarittime e nelle aree adiacenti verificatisi nel 1969. 
TABBLLA 15

\begin{tabular}{|c|c|c|c|c|c|c|c|c|}
\hline \multicolumn{2}{|c|}{ I) $A^{\prime} \Gamma A$} & \multicolumn{3}{|c|}{ GCT } & $\begin{array}{l}\text { lat. } \\
\text { N }\end{array}$ & $\begin{array}{c}\text { Joong. } \\
\text { l: }\end{array}$ & $\begin{array}{c}\text { l'rof. } \\
\text { h. } \\
\text { kill }\end{array}$ & $W_{\mathrm{L}}$ \\
\hline Jan & 7,1969 & 11 & 23 & 42,0 & $44^{\circ} 25^{\prime}$ & $7^{0} 16^{\prime}$ & 6.1 & 2,31 \\
\hline Jan & 26 & 04 & 58 & 03,3 & $44^{\circ} 22^{\prime}$ & 7015 & 6.0 & 2,57 \\
\hline Feb & 5 & 12 & 29 & 08,8 & $44^{\circ}+8^{\prime}$ & $7011^{\prime}$ & 5.3 & 3,03 \\
\hline Fel): & 28 & 20 & 37 & 58,8 & $44025^{\prime}$ & $7^{\circ} 14^{\prime}$ & 20.8 & 3,08 \\
\hline Mar & 17 & 18 & 39 & 43,0 & $44^{\circ} 16^{\prime}$ & $7025^{\prime}$ & 3.8 & 2,32 \\
\hline Mar & 18 & 01 & 09 & 51,3 & $44^{\circ} 33^{\prime}$ & $7^{\circ} 12^{\prime}$ & (i. 2 & 2,39 \\
\hline Apr & 12 & 05 & 13 & 44,0 & $44^{\circ} 26^{\prime}$ & $7^{\circ} 04^{\prime}$ & 5.5 & 2,56 \\
\hline Apr & 16 & 22 & 41 & 54,0 & $44^{\circ} 19^{\prime}$ & $7^{\circ} 18^{\prime}$ & 8.3 & 3,04 \\
\hline$\Lambda_{\mathrm{pr}}:$ & 28 & 15 & 20 & 28,0 & $44^{\circ} 03^{\prime}$ & $7^{\circ} 13^{\prime}$ & 9.1 & 2,64 \\
\hline May & 3 & 22 & 22 & 11,1 & $44^{\circ}+2^{\prime}$ & $7010^{\prime}$ & 6.8 & 2,600 \\
\hline May & 7 & 03 & 33 & 21,0 & $44^{\circ} 14^{\prime}$ & $7024^{\prime}$ & 3.1 & 1,93 \\
\hline$M a y$ & 17 & 10 & 58 & $5(i, 3$ & $43^{\circ}+9^{\prime}$ & $7038^{\prime}$ & 5.3 & 2,76 \\
\hline JuIn: & 21 & 23 & 33 & 31,2 & $44^{\circ} 10^{\prime}$ & $\left(0^{\circ} 59^{\prime}\right.$ & 15.1 & 2.29 \\
\hline Jul & 2 & 14 & 28 & 24,0 & $44^{\circ} 13^{\prime}$ & $80166^{\prime}$ & 7.1 & 2,90 \\
\hline Aur & 4 & 07 & 51 & 17,3 & $43059^{\prime}$ & $8^{0}(1) 7^{\prime}$ & 5.1 & 2,50 \\
\hline Aug & 15 & $0 \overline{5}$ & $60 ;$ & 04,4 & $44^{\circ} 34^{\prime}$ & $7011^{\prime}$ & 8.3 & 2,33 \\
\hline Oet & 4 & 10 & 20 & 56,8 & $44^{\circ} 12^{\prime}$ & $7034^{\prime}$ & 2.7 & 2,27 \\
\hline Oct & 8 & 02 & 55 & 42,4 & $44^{\circ} 19^{\prime}$ & $7020^{\prime}$ & 10.3 & 2,34 \\
\hline Oct & 11 & 17 & 10 & 44,8 & $43^{\circ} 56 i^{\prime}$ & $7^{\circ} 13^{\prime}$ & 8.0 & 2.28 \\
\hline Nov & 2 & 23 & 27 & 33,8 & $44^{0} 15^{\prime}$ & $7026^{\prime}$ & 5.9 & 2,55 \\
\hline Nov & 17 & 12 & 01 & 01,4 & $44^{\circ} 34^{\prime}$ & $6057^{\prime}$ & 6.1 & 3,01 \\
\hline Nov: & 22 & 07 & 49 & 14,4 & $44^{\circ} 2 t^{\prime}$ & $\left(j^{\circ}+9^{\prime}\right.$ & 7.6 & 3,52 \\
\hline
\end{tabular}

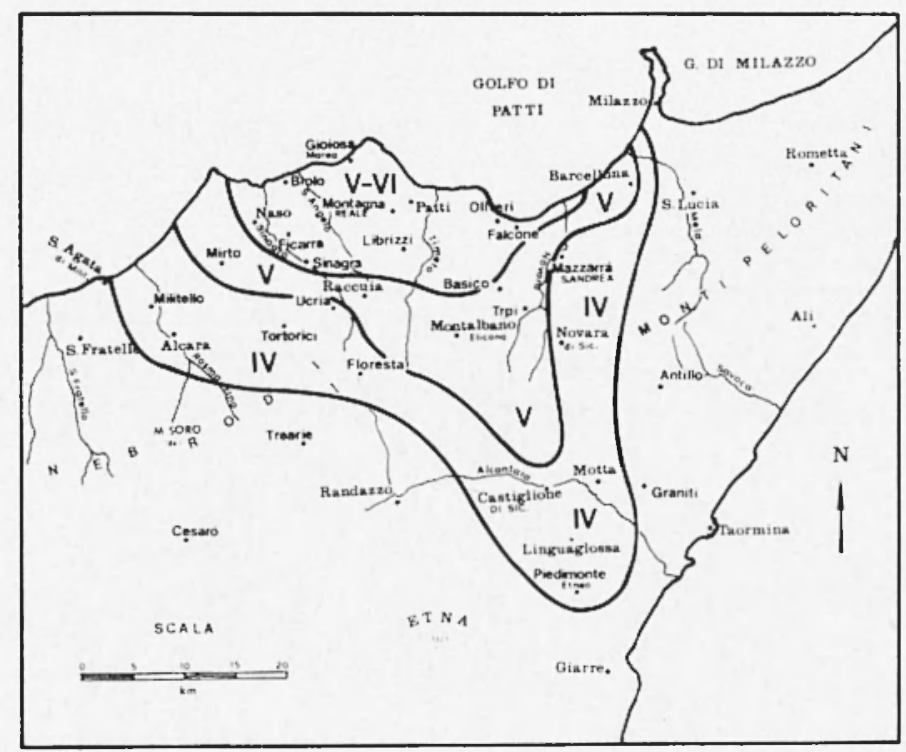

Fig. 17 - Isosiste del terremoto del Golfo di Pali, 30 Marzo 1969. J'area epicentrale è costituita prevalentemente da rocee magmatiche e metamorfiche. 


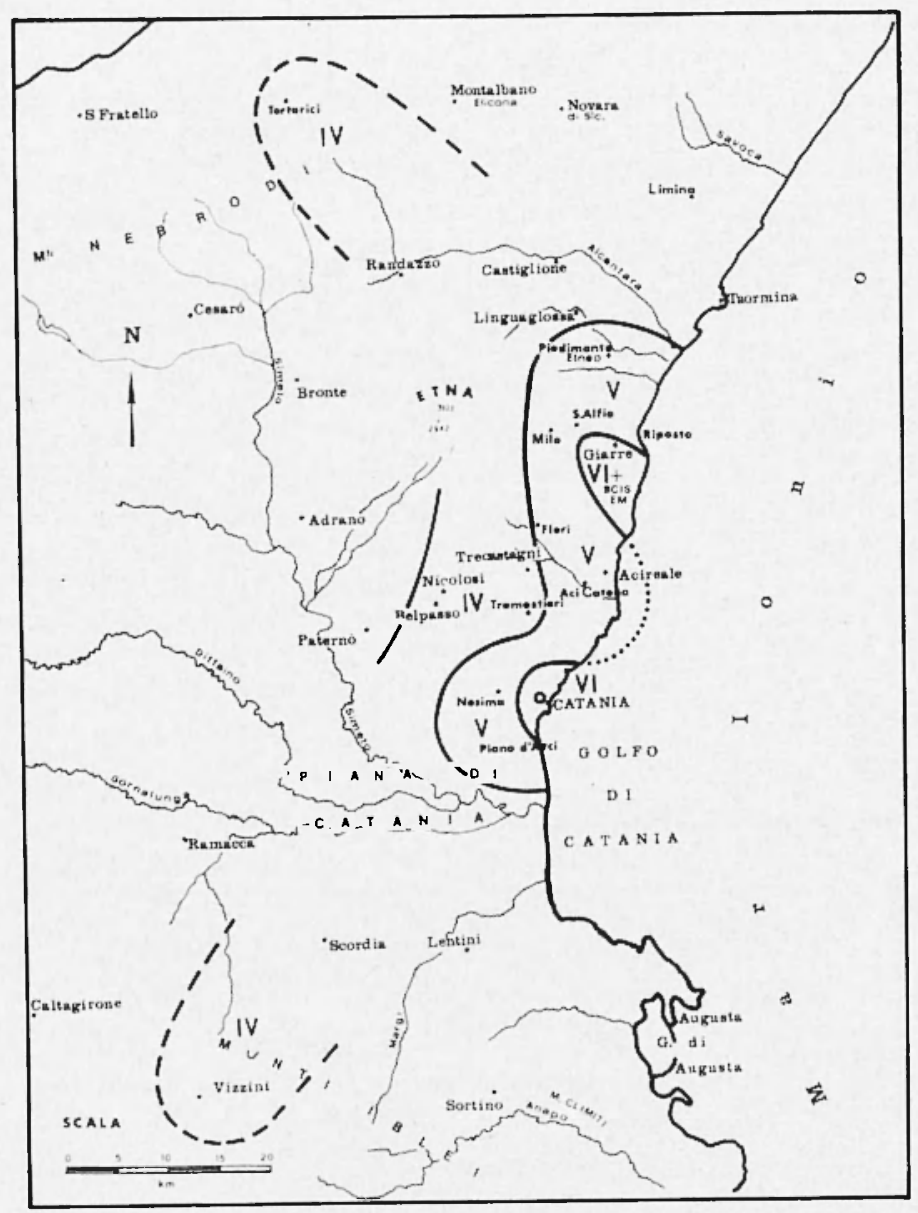

Fig. 18 - Isosiste del terremoto dell'Etna, 31 Marzo 1969. L'area in cui il sisma si è maggiormente manifestato, è costituita da rocee piroclastiche basiche. 


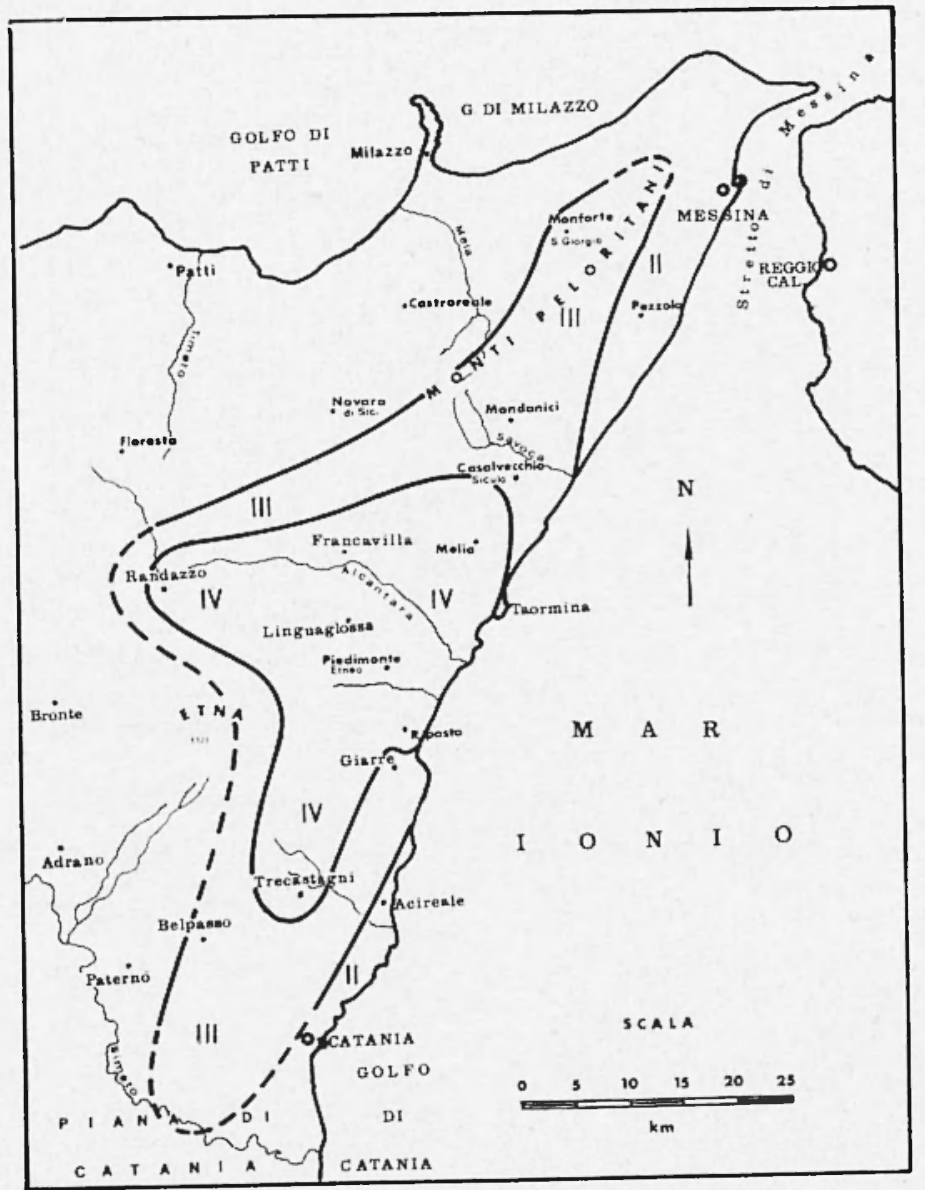

Fig. 19 - Isosiste del terremoto dell'Etna, 15 Aprile 1969. Liarea epicentrale ha interessato prevalentemente le rocee costituenti l'apparato vuleanico etneo. 


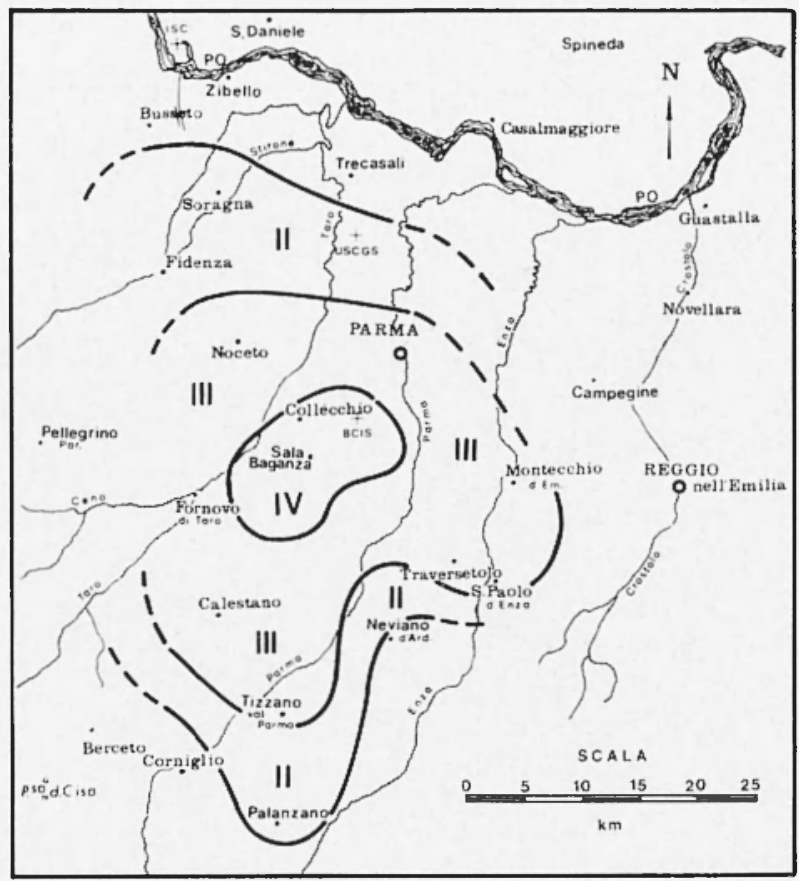

Fig. 20 - Isosiste del terremoto della Val Baganza, 24 Giugno 1969.

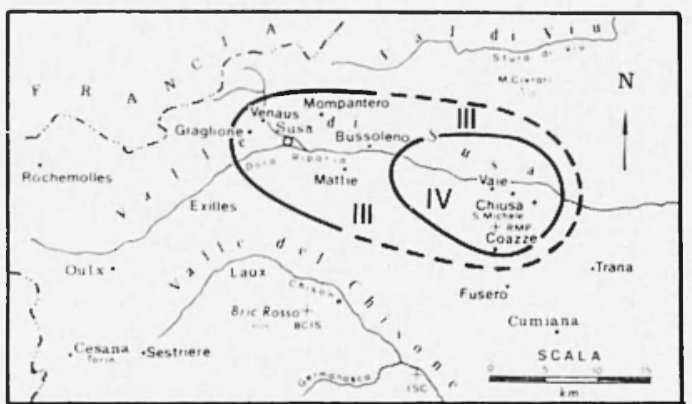

Fig. 21 - Isosiste del terremoto della Valle di Susa, 2 Luglio 1969. 


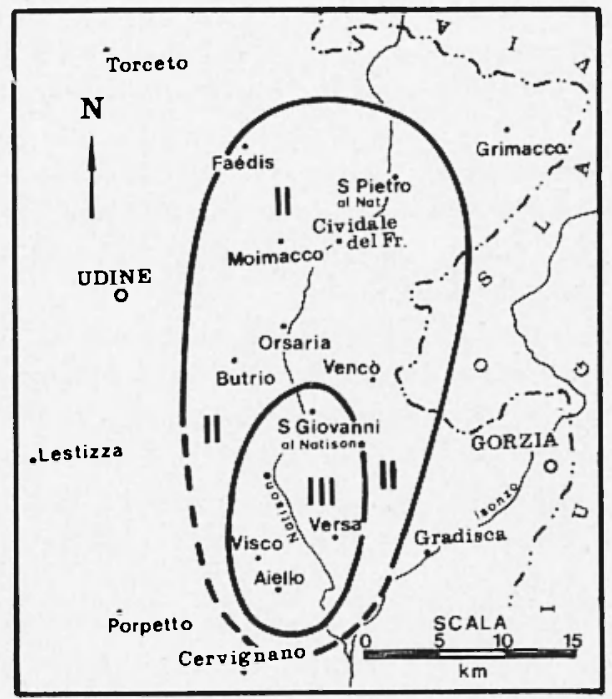

Fig. 22 - Isosiste del terremoto del Friuli, 13 Agosto 1969. L'area epicentrale è compresa entro le formazioni alluvionali pleistoceniche.

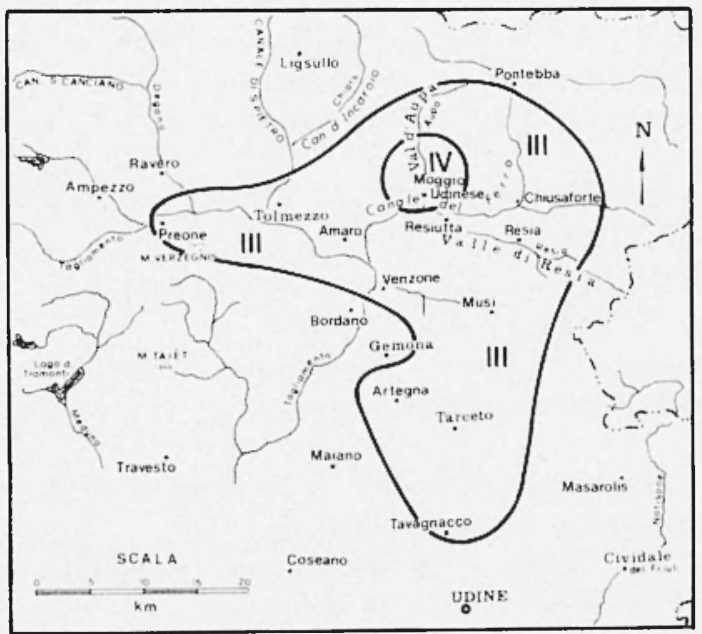

Fir. 23 - Isosiste del terremoto della Carnia, 11 Dicembre 1969. 


\section{BIBIJOGRAFIA}

(1) Accordi B., Axgerucci A., Sinxa G., 1967. - Note illustrative della Carta geologica d'Italia - joglio Frosinone-Cassino. Polig. dello Stato, Roma.

(2) Aureiro V., Console R., Gasparini C., 1973. - La Magnitudo calcolata mediante sismografi a breve periodo. "Annali di Geofisica", XXVI, 4.

(3) Bontolotт V., 1966. - La tettonica trasversale dell'Appennino. "Boll. Soc. Grol. Ital. ", LXXXV.

(4) Bossolasco M., Eva C., 1971. - The seismicity in marilime Alps and adjacent areas. Comm. Seism. Europ. - XII Assemblea Generale, 1970.

${ }^{5}{ }^{5}$ BYERLY P', 1928. - The nature of the first motion in the chilean earthquale of November 11, 1922. "American journal Science", 5n sez., XVI, 93.

$\left.{ }^{6}{ }^{6}\right)$ BYERLY P., 1938. - The earthquake of July 6, 1934-Amplitudes and first motion. "Bull. Seism. Soc. of Amer. ", 28.

$\left({ }^{7}\right)$ Byerty P', Widian Y., Stauder S. J., 1969. - Motion at the source of an earthquake. Publications of the Dominion Observatory.

${ }^{8}$ ) ColacicciI R., 1966. - Caratteristiche della facies abruzzese alla luce delle moderne indagini geologiche. "Mcm. Soc. Geol. Ital.", V, 1.

$\left({ }^{9}\right)$ Console R., Peronaci F., Sonatidia A., 1973. - Relazioni sui jenomeni sismici dell'anconitano (1972). Monografia suppl. "Annali di Geofisica", XXVI.

${ }^{(10)}$ De Paxfitis M., 1969. - Un periodo sismico nella zona dei Monti della Tolfa. "Annali di Geofisica ", XXII, 3.

(11) Di Fiı.ıpo D., 1950. - Sulla rappresentazione in superficie della natura dinamica di una scossa allipocentro. "Annali di Ocofisica ", III, 2.

${ }^{\left({ }^{12}\right)}$ DI FILIPPO D)., 1950. - Sulla rappresentazione in superficie della natura dinamica di una scossa con ipocentro profondo. "Annali di Geofisica ", III, 3.

${ }^{(13)}$ Fazzini P., ed al., 1972. - Cieologia dei IIonti della Tolfa. "Mem. Soc. Geol. Ital. ", XI, 1.

(14) Gasparini C., Giovani L., 1973. - Attività sismica in Italia nel 1970. "Annali di Geofisica ", XXVI, 2-3.

${ }^{(15)}$ KEYLIS BoROK V. I., 1957. - The determination of earthquake mechanism using both longiludinal and trasversal waves. "Annali di Geofisiea", $\mathrm{X}, \mathbf{1}-\mathbf{2}$.

${ }^{(16)}$ Ianuro C., Negretti G., 1969. - Il vulcanismo nella tuscia Romana. C.N.R. Quaderno n. 5 - Roma.

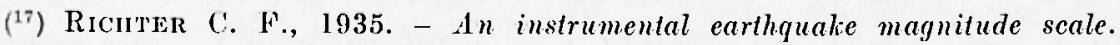
"Bull. of the Seismol. Soc. of Am.", I.

$\left.{ }^{18}\right)$ Rosini E., 1940. - Il terremoto della Garjagnana del 15 Ottobre 1939. "Ia Ricerca Scientifica ", XI, 7-8. 


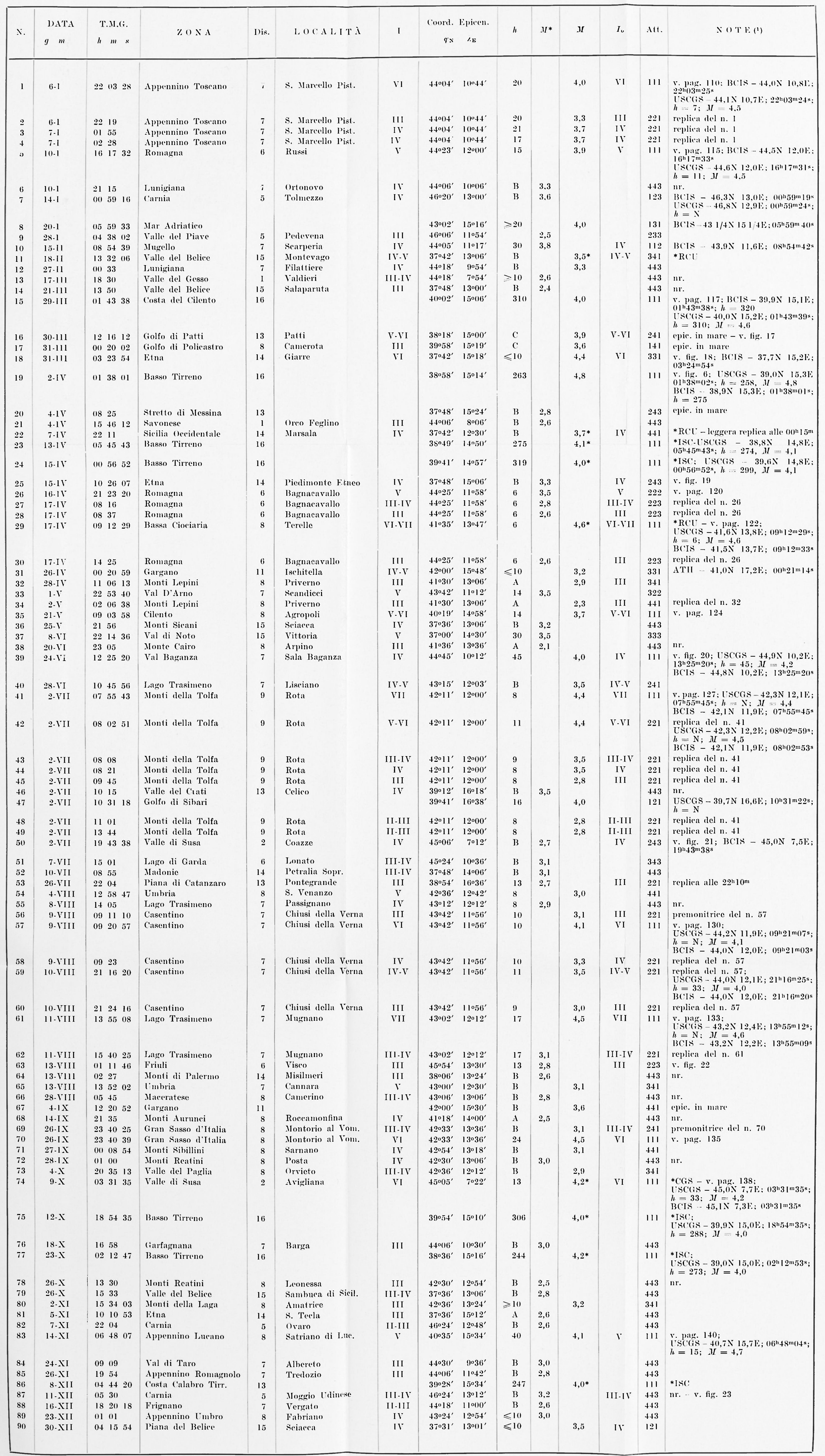

(1) La magnitudo strumentale s'intende sempre caleolata con le registrazioni effettnate presso l'Osservatorio di Roma Monte Porzio, altrimenti l'asteriseo richiama la sigla dell'organizzazione

nr. = indica che l'evento non ha dato luogo a registrazione sismograficia 